\title{
Influences of Models on the Unsteady Pressure Characteristics of the NASA National Transonic Facility (invited)
}

\author{
Gregory Jones ${ }^{1}$, Sundareswara Balakrishna ${ }^{2}$, Joshua DeMoss ${ }^{3}$, Scott Goodliff $^{4}$, Matthew Bailey ${ }^{5}$ \\ NASA Langley Research Center, Hampton, VA, 23681
}

\begin{abstract}
Pressure fluctuations have been measured over the course of several tests in the National Transonic Facility to study unsteady phenomenon both with and without the influence of a model. Broadband spectral analysis will be used to characterize the length scales of the tunnel. Special attention will be given to the large-scale, low frequency data that influences the Mach number and force and moment variability. This paper will also discuss the significance of the vorticity and sound fields that can be related to the Common Research Model and will also highlight the comparisons to an empty tunnel configuration. The effectiveness of vortex generators placed at the interface of the test section and wind tunnel diffuser showed promise in reducing the empty tunnel unsteadiness, however, the vortex generators were ineffective in the presence of a model.
\end{abstract}

\section{Nomenclature}

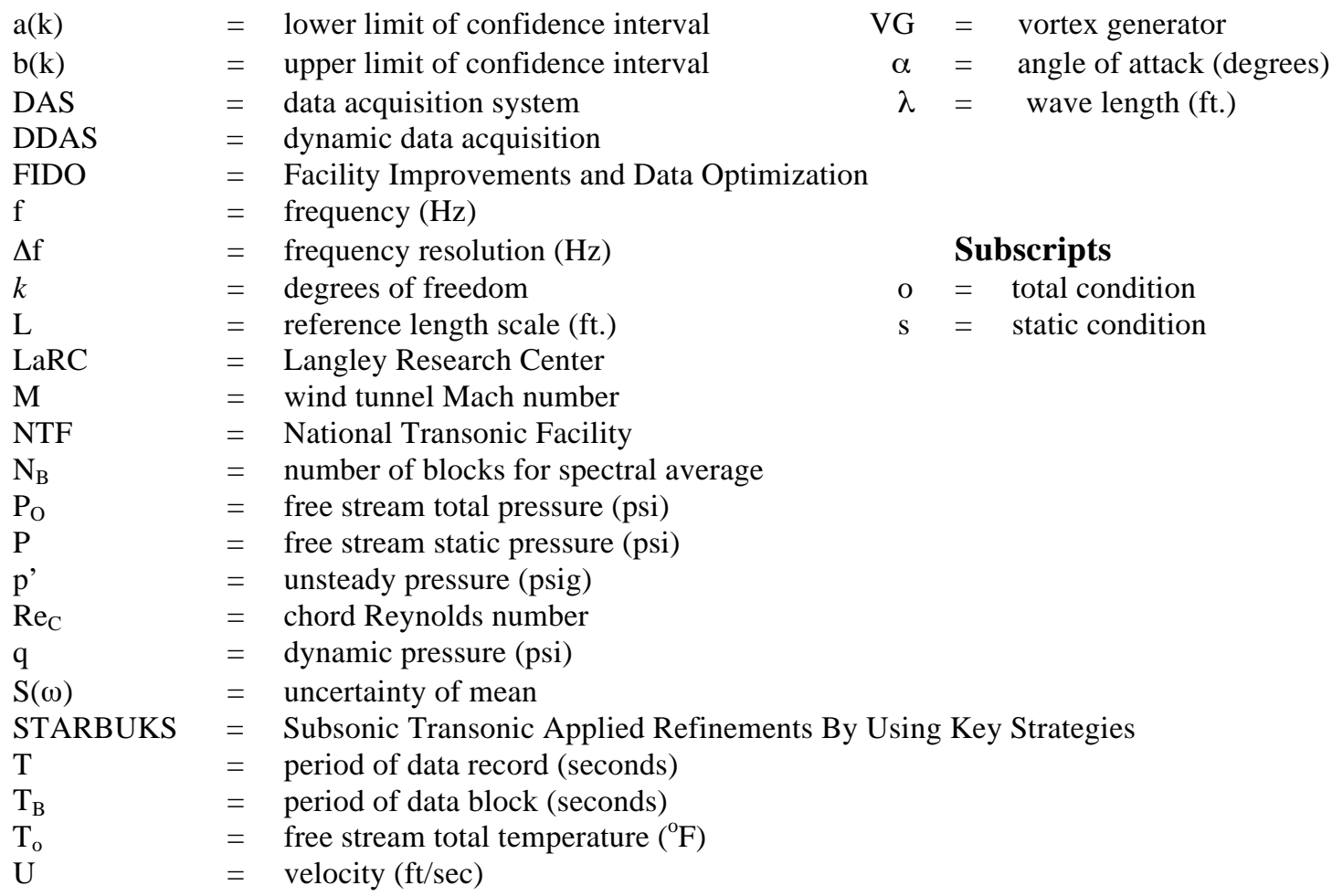

\footnotetext{
${ }^{1}$ Research Engineer, Configuration Aerodynamic Branch, Mail Stop 267, AIAA Senior Member

${ }^{2}$ Research Engineer, Vigyan, Mail Stop 274

${ }^{3}$ Test Engineer, Analytical Services \& Materials, Mail Stop 267

${ }^{4}$ Test Engineer, Jacobs Technology, Inc., Mail Stop 267, AIAA Senior Member

${ }^{5}$ Test Engineer, Jacobs Technology, Inc., Mail Stop 267, AIAA Member
} 


\section{Introduction}

$\mathrm{R}$ ecent modifications to the NASA Langley Research Center National Transonic Facility (NTF) shown in Figure 1, have prompted a characterization of the test section flow field that includes the unsteady pressure field and the flow uniformity. The current and future testing demands of this facility require an updated assessment of the flow quality for the purposes of testing advanced technologies at relevant operating conditions. Previous investigations ${ }^{1,23,4,5}$ of the unsteady characteristics of NTF have emphasized free stream hot-wire and microphone data.

This paper will highlight three tunnel entries that focus on several potential tunnel modifications that included vortex generators in the high speed diffuser, and a second throat used to

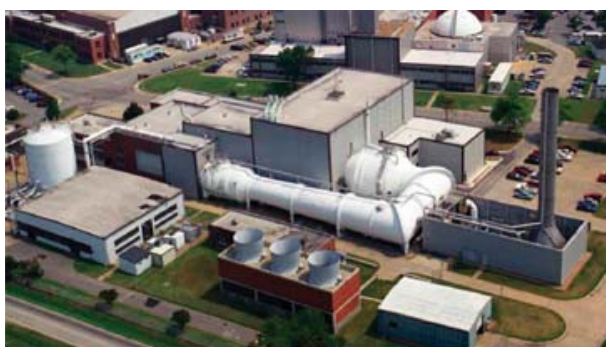

Figure 1. Aerial Photo of the NTF. create a choked condition downstream of the test section. The emphasis of this evaluation will attempt to associate the cause and effects related to the influence of these modifications on the unsteady pressure fields and tunnel dynamics on the model. These data will also highlight the coherent and random nature of the measured unsteady forces and moments as well as unsteady pressures that influence data acquisition periods and data rates needed to minimize uncertainty and improve data repeatability. A summary of how any of these disturbances influence the flow field on a model can be found in reference 6.

Before one can begin to evaluate the perturbations in a generalized flow field, it is necessary to understand the potential sources of the perturbations. In general, the unsteady characteristics of a transonic wind tunnel can be divided into three modes, vorticity, entropy, and $\operatorname{sound}^{7,8,9}$. These three modes cannot be measured directly but can be related to the measured quantities of velocity, density, and total temperature fluctuations ${ }^{10}$. While these characteristics are used to define the general fluctuating flow field, it is difficult for the experimentalist to measure them without some knowledge of the sound mode ${ }^{11,12}$. The fluctuating pressure field is typically related to the sound field, however, it is also a function of the fluctuating velocity and temperature fields, namely vorticity and entropy. These flow fields typically have different length scales and travel at different speeds, so is difficult to resolve these measurements in the reverberant environment of a transonic wind tunnel. Acoustic waves travel in all directions at the speed of sound minus the local velocity while the vorticity and entropy travel along streamlines at the transport velocity of the local flow. One can estimate the influence of a model by subtracting the empty tunnel spectra from the tunnel spectra with the model located in the test section. Some sources of the vorticity, entropy, and sound are shown in Figure 2, and include fan and fan interactions, turning vanes, the heat exchanger, anti-turbulence screens,

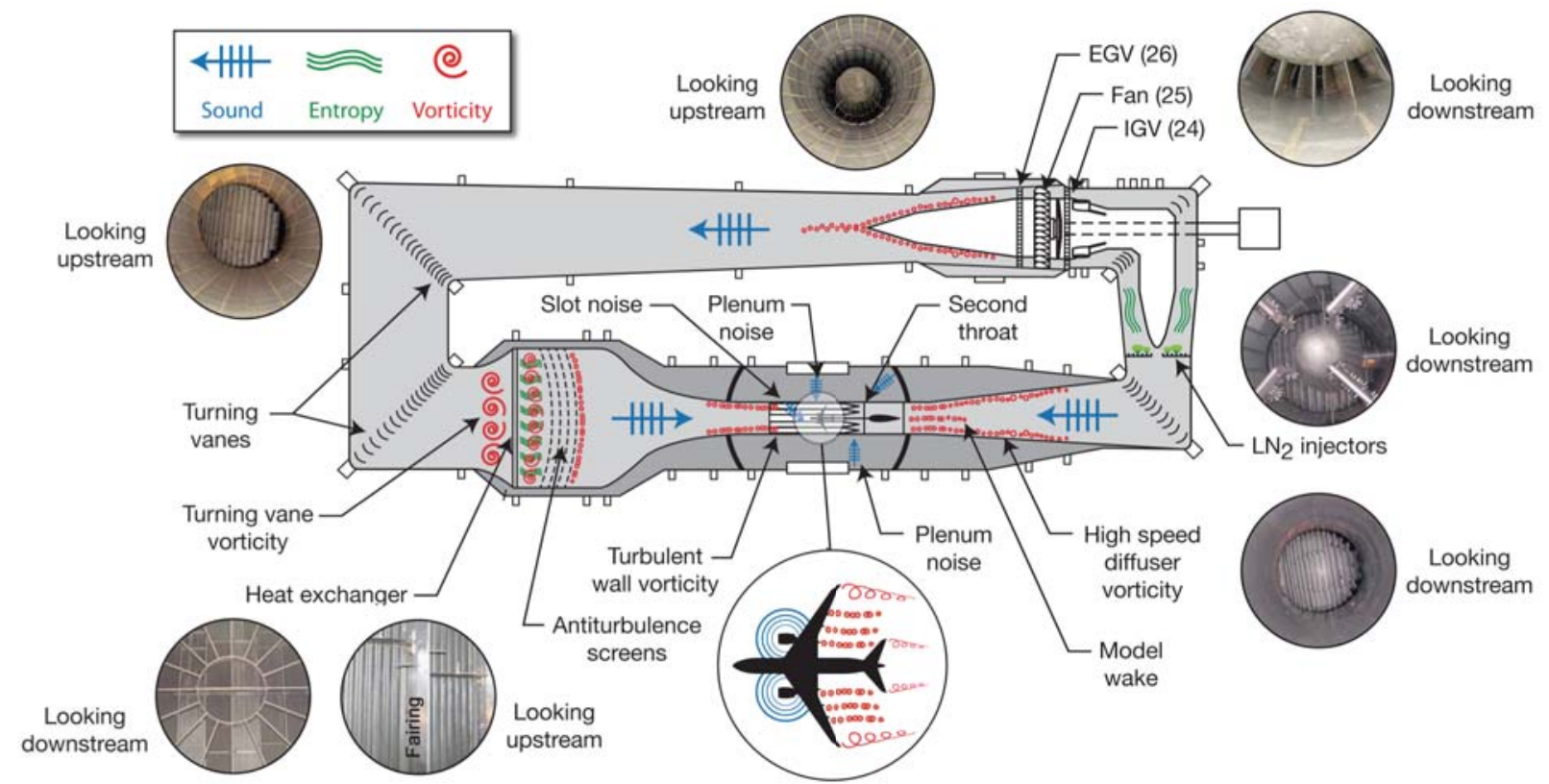

Figure 2. Sketch of a transonic wind tunnel and potential sources of disturbances. 
liquid nitrogen $\left(\mathrm{LN}_{2}\right)$ injectors, plenum resonance, circuit boundary layers, and the interactions with the model and model support system. The influence of any of these wind tunnel components depends on the efficiency of the turbulence manipulators and the propagation of a disturbance into the test section. Identification of these sources becomes difficult due to the scales, mixing, and interactions of the sources with each other. Some of the wind tunnel components identified above create broadband small-scale disturbances (e.g., turbulent wakes and boundary layers) while others generate coherent disturbances (e.g., fan interactions). Spectral analysis techniques will be used in an attempt to identify the influence of the different disturbances on the test section unsteady pressure field.

\section{Experimental Setup}

\section{A. Wind Tunnel}

The $\mathrm{NTF}^{13}$ (Figure 3) is one of a limited number of wind tunnel facilities that can achieve flight Reynolds numbers and Mach numbers for transport type aircraft for both cruise and high lift operations. The tunnel is a fan-driven, closed-circuit, continuous-flow, pressurized wind tunnel capable of operating either in dry air at warm temperatures or in nitrogen gas from warm to cryogenic temperatures. The test section is $8.2 \mathrm{ft}$. by 8.2 $\mathrm{ft}$. in cross section and $25 \mathrm{ft}$. in length. The test section floor and ceiling are slotted (6 percent open), and the sidewalls are solid. The wind tunnel is capable of an absolute pressure range from 1 atmosphere to 8.3

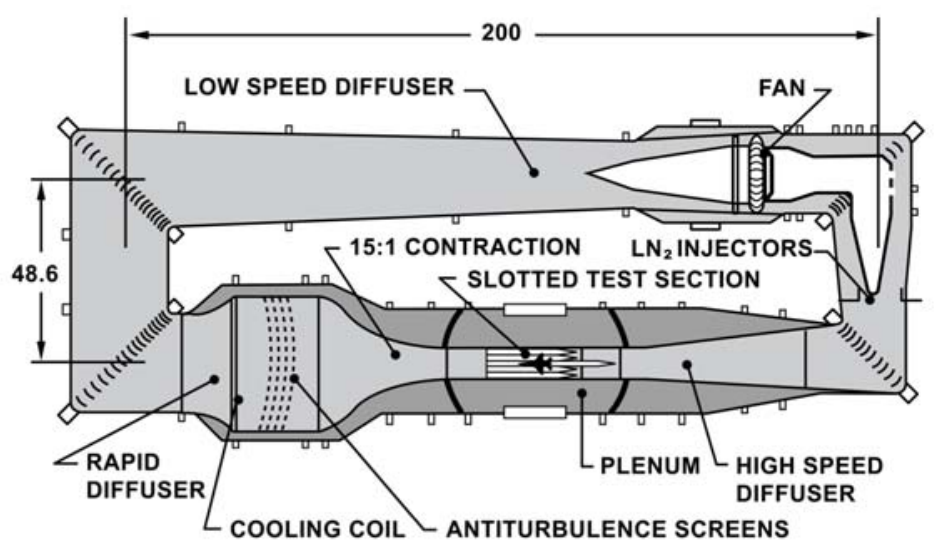

Figure 3. Sketch of the NTF. (All linear dimensions are in feet). atmospheres, a temperature range from $-250^{\circ} \mathrm{F}$ to $130^{\circ} \mathrm{F}$, a Mach number range from 0.1 to 1.2 , and a maximum unity Reynolds number of $146 \times 10^{6}$ per foot at Mach 1 .

Mach number control above Mach 0.80 has been investigated at NTF for a number of years, and data analysis has shown that a correlation exists between Mach number variability and drag coefficient variability for some models. Consequently, it is hypothesized that if Mach variability can be reduced, drag repeatability will improve. The two concepts that will be described in this paper focus on a second throat configuration and a vortex generator configuration.

Re-entry flaps are typically used to optimize the mixing of the flow from the plenum and test section boundary layers into the high-speed diffuser. For this series of tests a combination of re-entry flaps and movable wall settings were used to setup a second throat or minimum to create a choked condition at station 25 feet, just beyond the exit of the test section. Figure 4 highlights a typical baseline and choked tunnel configuration.

Vortex generators (VGs) are commonly used in wind tunnels to delay boundary-layer separation in the high
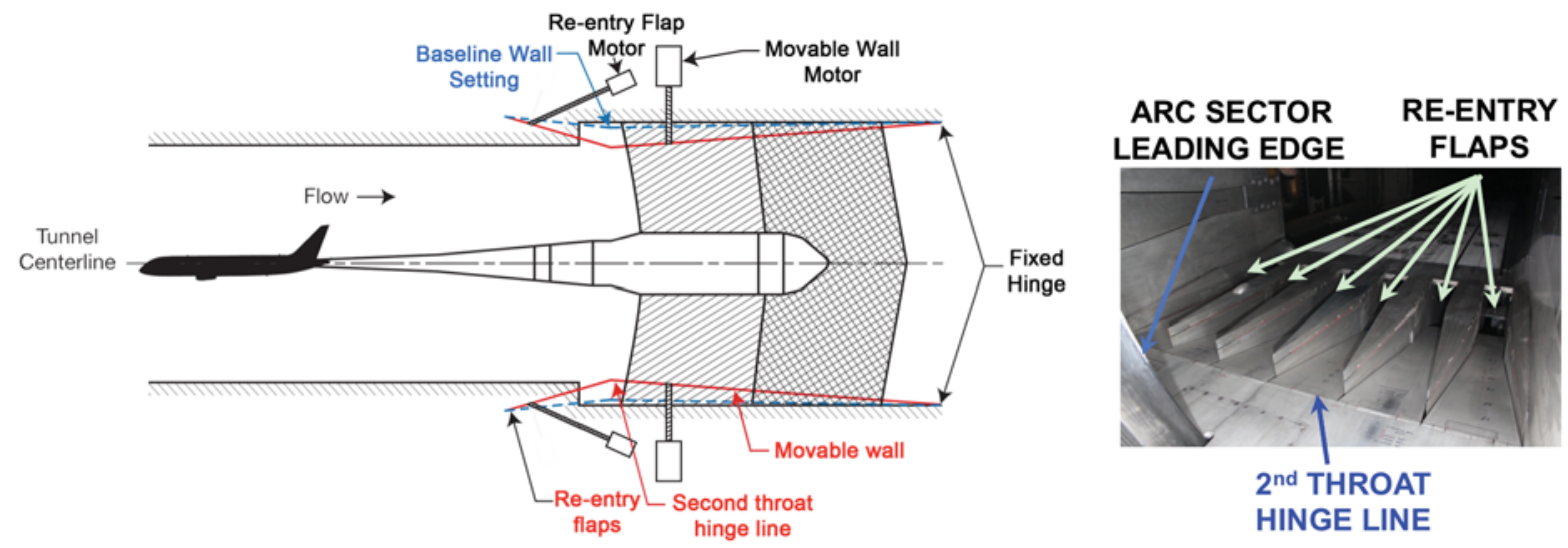

Figure 4. Re-entry flap and movable wall configuration forms the second throat at the 25 foot station. 
speed diffuser. The vane-type vortex generator configuration being evaluated in this paper is shown in Figure 5. Four pairs of counter-rotating trapazodial VGs were attached to each wall downstream of the second throat pivot point. Three VG configurations were tested: floor and ceiling VGs, sidewall VGs, and a combiation of floor/ceiling VGs and sidewall VGs.

\section{B. Instrumentation}

There were two independent data acquisition systems utilized for this series of tests, the NTF Data

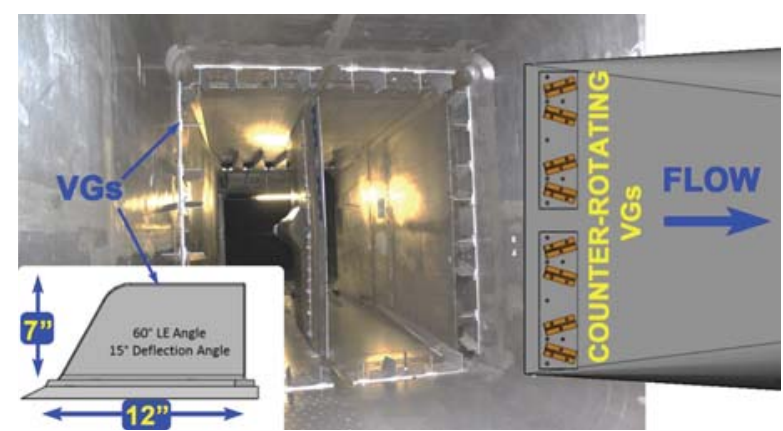

Figure 5. Floor and ceiling VG configuration. Acquisition System (DAS) and the NTF Dynamic Data Acquisition System (DDAS). The emphasis of this paper will be on data collected by the DDAS system. The primary components of this system consisted of a Pacific 6120 amplifier and filter system, and a National Instruments high-speed 24-bit A/D system that can sample up to 204.8kHz. This 32-channel system was configured to simultaneously acquire seven unsteady pressure transducers and six force and moment balance channels. A schematic of the DDAS data stream is shown in Figure 6 . Data acquisition on the DDAS was triggered from the DAS so that both systems were synchronized to start and stop acquisition at the same time. All of the DAS and DDAS data were post-processed from files that were stored on the

$12.8 \mathrm{kHz}<$ Sample Rate $<102.4 \mathrm{kHz}$

(12.8k Samp/Sec Typical)

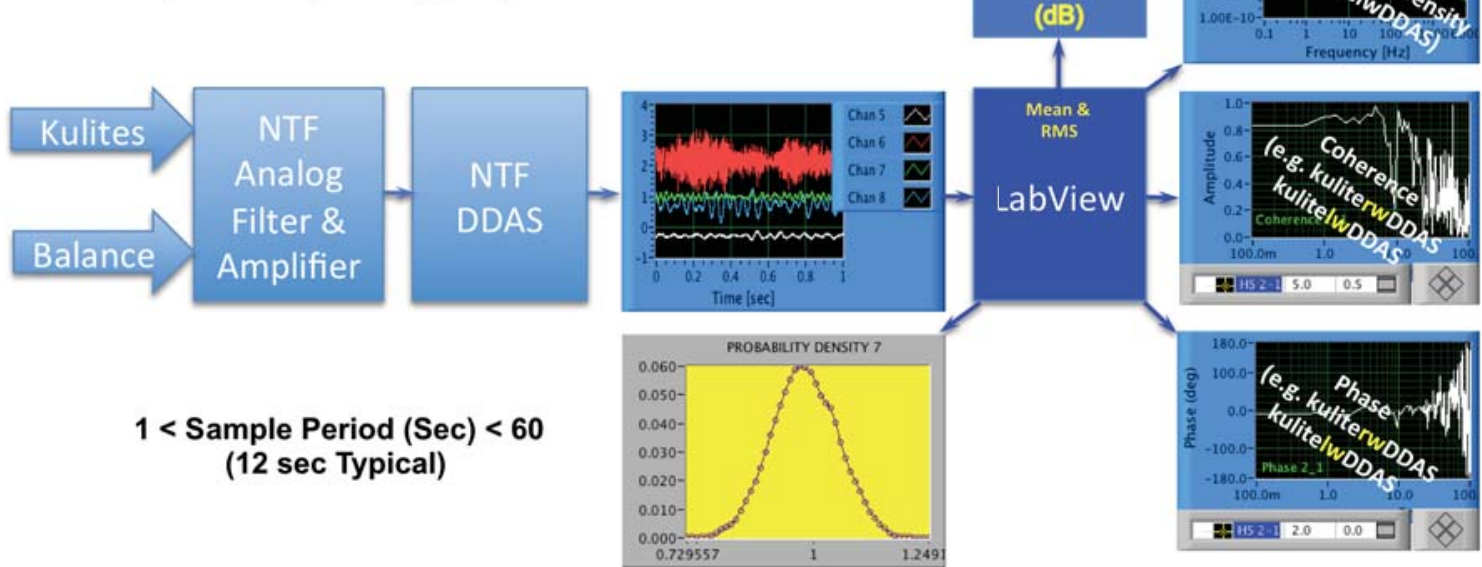

Figure 6. Schematic of NTF Dynamic Data Acquisition System.

LaRC AeroCompass system.

The following discussion serves to highlight the different approaches to evaluating the unsteady characteristics of the pressure and balance data described throughout this paper. In general, it is important to discuss the average or mean quanities separately from the unsteady quantities as many time signals are not easily analyzed in the time domain. While the mean and standard deviation values can be obtained from a statistical average, the underlying character of the time histories are often unintelligible. One can expand these time histories into the frequency domain through the use of Fast Fourier Transformation. ${ }^{14}$ Since the time and frequency domain representations contain precisely the same information in the sense that one may be recovered from the other by integration, the generation and potential effects of a signal may often be more easily understood in the frequency domain. The characterization of any measured parameter (e.g., pressure or balance data) will depend on the bandwidth of interest.

Throughout this series of tests, the spectral analysis focused on the power spectral density and the coherence features of the data. Hanning windows are applied to the computed power spectral densities with a frequency resolution between $0.03 \mathrm{~Hz}$ to $10 \mathrm{~Hz}$. Length scale characteristics were also obtained by evaluating the wavelength given by:

$$
\lambda=\text { Wavelength }=\text { Local Velocity/Frequency }
$$


Typical acquisition periods for any given point lasted for a minimum of 12 seconds, and sample rates varied between 12,800 and 102,400 samples per second for the DDAS and a constant 400 samples per second for the DAS. Kulite signals were directly interfaced to the DDAS, but the balance signals were isolated thru the DAS A/D system prior to the DDAS connections. There were also several opportunities to collect long sample periods of data in order to analyze the low frequency characteristics of the tunnel. These long data points were composed of 30 second, 60 second, and 120 second acquisition periods.

In general, the uncertainty of the spectral data can be related to the degrees of freedom given by:

$$
K=2 \mathrm{~N}_{\mathrm{B}}
$$

where the total period is divided into $\mathrm{N}_{\mathrm{B}}$ blocks of data. The confidence interval of the spectra is a function of a chisquare random variable and is directly related to the number of averages $\left(\mathrm{N}_{B}\right)$ shown in Figure 7. The variation of a chi-square random variable described in Figure 7 represents $80 \%$ of the values taken by the random variable to lie between the bounds shown. Thus, these bounds represent the $80 \%$ confidence limits on the random variable. As the number of blocks increases the reduction in variability reduces the uncertainty of the estimate. Of course, this reduction in variability has not been achieved without cost. Note that the limit of the bandwidth of the spectral estimate is given by:

$$
\Delta f=1 / \mathrm{T}
$$

If the data are broken into blocks, the effective data length is no longer $T$, but $T_{B}$. Thus, the effective bandwidth of the estimate $\Delta f$ has increased to:

$$
\Delta f=1 / \mathrm{T}_{\mathrm{B}}
$$

In order to reduce the variability, the resolution has also been reduced. Writing $k=2 \mathrm{~N}_{\mathrm{B}}=2 \mathrm{~T} / \mathrm{T}_{\mathrm{B}}$ yields the fundamental relation:

$$
k=2 \Delta f \mathrm{~T}
$$

That is, the degrees of freedom are equal to twice the bandwidth in hertz times the data length. Thus, if $\mathrm{T}$ is fixed, a tradeoff of reduced variability or reduced resolution becomes apparent. The only way out of this predicament is to obtain more data, by increasing the sampling period $\mathrm{T}$. This tradeoff is illustrated in Table 1 where a fixed confidence interval can be achieved for a 12 second period or a 120 second period by

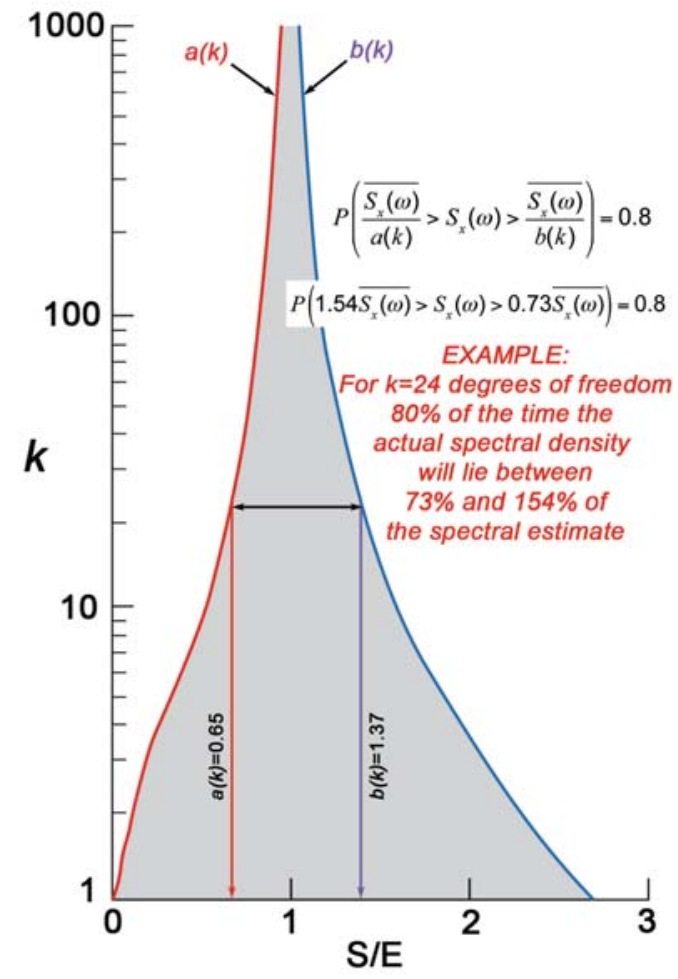

Figure 7. Variation of Chi-square random variable for $80 \%$ confidence interval.

\begin{tabular}{|c|c|c|c|c|c|c|c|}
\hline $\begin{array}{c}\text { Total Period } \\
\mathrm{T}(\mathrm{Sec})\end{array}$ & $\begin{array}{c}\text { \# of Blocks } \\
\mathrm{N}_{\mathrm{B}}\end{array}$ & $\begin{array}{c}\text { Block Period } \\
\mathrm{T}_{\mathrm{B}}(\mathrm{Sec})\end{array}$ & $\begin{array}{c}\text { Frequency } \\
\text { Resolution } \\
\Delta \mathbf{f}(\mathrm{Hz})\end{array}$ & $\begin{array}{c}\text { Degrees } \\
\text { of Freedom } \\
(\mathbf{k})\end{array}$ & $\begin{array}{c}\text { Left Boundary } \\
\text { Chi-Square } \\
\text { Estimate }\end{array}$ & $\begin{array}{c}\text { Right Boundary } \\
\text { Chi-square } \\
\text { Estimate }\end{array}$ & $\begin{array}{c}\text { Spectral } \\
\text { Density } \\
\text { Variability }(\%)\end{array}$ \\
\hline 12 & 120 & 0.1 & 10 & 240 & 0.88 & 1.12 & $89-114$ \\
\hline 12 & 12 & 1 & 1 & 24 & 0.65 & 1.37 & $73-154$ \\
\hline 60 & 60 & 1 & 1 & 120 & 0.78 & 1.15 & $87-128$ \\
\hline 60 & 6 & 10 & 0.1 & 12 & 0.55 & 1.55 & $64-182$ \\
\hline 120 & 12 & 1 & 1 & 240 & 0.88 & 1.12 & $89-114$ \\
\hline 120 & 12 & 10 & 0.1 & 24 & 0.65 & 1.37 & $73-154$ \\
\hline 120 & 4 & 30 & 0.033 & 8 & 0.45 & 1.65 & $61-222$ \\
\hline
\end{tabular}

Table 1. Variability changes for different spectral sampling configurations. 
changing the block period or frequency resolution by a factor of 10 .

As previously stated, 12 second sample periods were typically acquired for both the DAS and DDAS systems. In general,u a $10 \mathrm{~Hz}$ resolution was used to capture the bandwidth necessary to characterize the unsteady flow characteristics of the tunnel $(10<$ Freq $(\mathrm{Hz})<20,000)$. The low frequency analysis focussed on bandwidths less than $10 \mathrm{~Hz}$ that required a resolution less than or equal to $1 \mathrm{~Hz}$. The low frequency analysis utilized long sampling periods of data to achieve good confidence intervals. The spectral confidence intervals for different sampling periods and block sizes used for this report are highlighted in Table 1.

\section{1) Unsteady Pressure Transducers}

The unsteady pressure measurements focused on two basic configurations that utilized flush mounted wall transducers and probe mounted transducers that were part of a 7-foot flow survey rake experiment. Kulite designed all of these differential piezo-resistive transducers for cryogenic conditions. Kulite model CCQ-062-25D transducers with B screens were used in the rake probes as shown in Figure 8 to measure fluctuating total $\left(\mathrm{Pt}^{\prime}\right)$ and static $\left(\mathrm{Ps}^{\prime}\right)$ pressure. The flush mounted wall transducers utilized the Kulite CCQ-093-5D and CT-190-5D models.

The frequency response of these configurations are influenced by several factors that include the natural frequency of the sensor, the size of the sensor, the mounting of the sensor, the signal conditioning, and the orientation to the unsteady pressure field. The manufacturer characterized the typical frequency response for the CCQ-062-25D and the CCQ-093-5D to be $250 \mathrm{kHz}$ and $150 \mathrm{kHz}$ respectively. Recent evidence ${ }^{15}$ has shown that the influence of the screens significantly reduces the frequency response of these transducers. This is exemplified in a transducer similar to the CCQ-062-25D where the frequency response is flat up to $37 \mathrm{kHz}$.

To protect the small differential gauges from pressures that approach 120 psia, special attention was given to the probe and wall mounted gauge references. In general the reference pressure was routed to a mean pressure that was consistent with the measurement side of the gauge. Ideally this would create a zero mean pressure across the gauge. For the wall mounted gauges the reference returned to a static tap located within 1 inch of the unsteady measurement location (see figure 9)

A tranfer function $\left(\mathrm{P}_{\mathrm{MEAS}} / \mathrm{P}_{\mathrm{REF}}\right)$ was used to determine the tubing response of the unsteady pressure system. A magnitude of one and phase shift of zero for any frequency being measured indicates that the pressure being measured at the probe location is being sensed without any loss or amplification at the transducer diaphram. In general, this is not the case for any pressure system that utilizes tubing. The theoretical Helmholtz resonance of the tubing and cavity of the unsteady pressure system was determined using an Acoustic Transfer Function code developed by AeroProbe ${ }^{16}$. The code is based on Bergh and Tijdeman experiments ${ }^{17}$ and is capable of analyzisng multiple tubing configurations as shown in Figure 10.

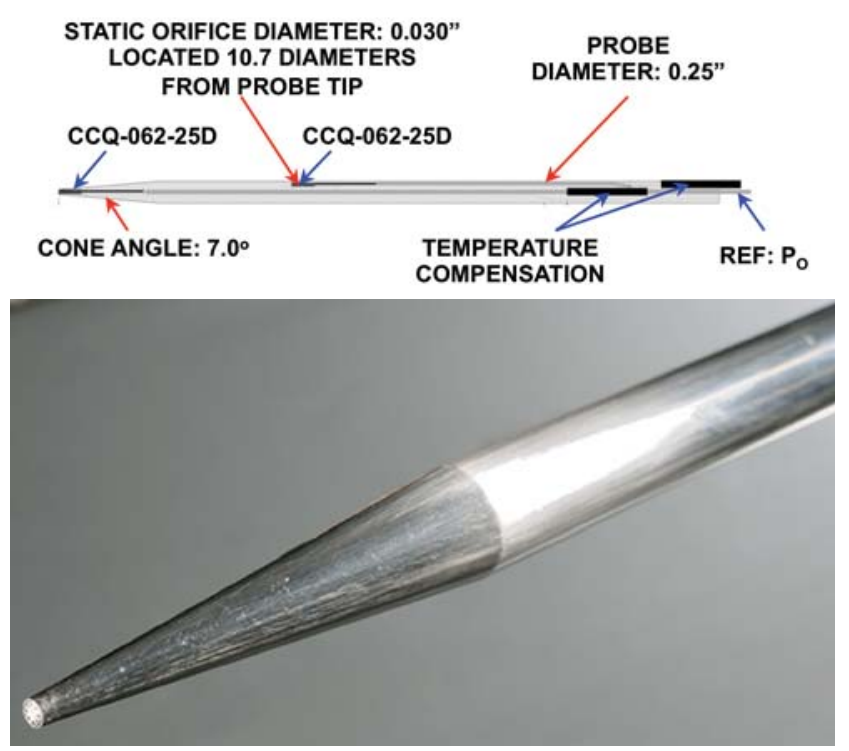

Figure 8. Probe configuration for the unsteady rake pitot-static probe.

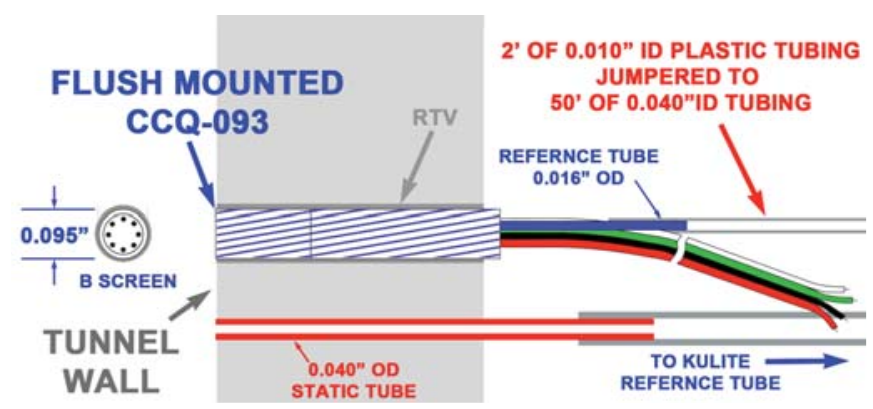

Figure 9. Wall mounted unsteady transducer with reference routed to nearby static pressure tap.

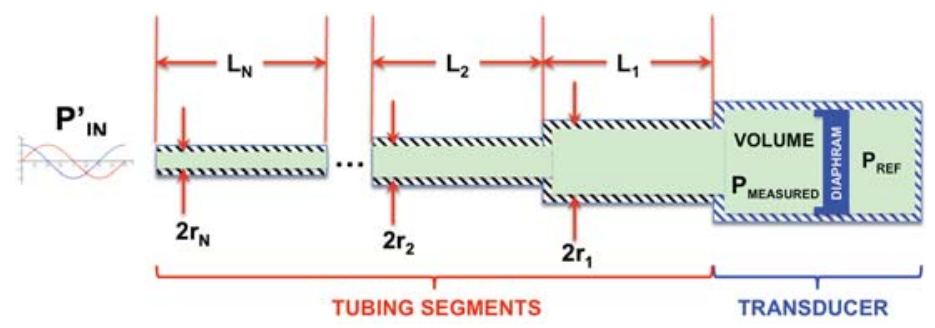

Figure 10. General multiple tubing configuration used to estimate system tubing response. 
To reduce the impact of the unsteady pressure being transmitted to the sensor through the reference tube, a pneumatic tubing filter was designed to attenuate frequencies above $1 \mathrm{~Hz}$. It was determined that 50 feet of 0.040 inch tubing would attenuate the high frequency reference signals as shown in Figure 11.

The only transducer that was not flush mounted for this series of tests was the static probe located in the rake. The measurment side of the transducer was mounted 90 degrees to the orifice location as shown in Figure 8. The 0.030 inch diameter orifice is coupled to a small cavity that is located in front of the transducer. Figure 12 highlights the estimated resonance response for this configuration to be at $42 \mathrm{kHz}$.

The temperature compensation provided by the manufacture was inadequate for the NTF applications so each transducer was calibrated over a range of temperatures that was consistent with the NTF tunnel conditions. An example of the variations in sensitivities for different transducers is shown in Figure 13. Since the focus of the Kulite data was just the unsteady portion of the signal, the off-set was ignored and the mean was subtracted from measured voltage.

\section{2) Mach Measurement System}

The Mach Measurement System (MMS) is based on simultaneous measurements obtained in the settling chamber and plenum with optional total pressure measurements obtained from a pitot tube located in the contraction as shown in Figure 14. The transducers for this system are optimized for the nine atmosphere pressure range of the facility. Two Fluke $7052 \mathrm{i}$ absolute gauges are used for total pressure measurements and two Fluke 7052i differential gauges are used for the static pressure measurements. These gauges are automatically selected based on the optimal pressure range needed for the measurement. The digital outputs of these gauges are low pass filtered at 1 hertz.

The MMS utilized the NTF DAS while the Kulite (and balance) data was acquired with the high speed DDAS. The bandwidth of the DAS and DDAS was significantly different (400 samples per second vs. 12800 (or 104000) samples per second, respectively). To make comparisons between the two systems, it was necessary to determine the similarities between them and to better understand their respective response characteristics.

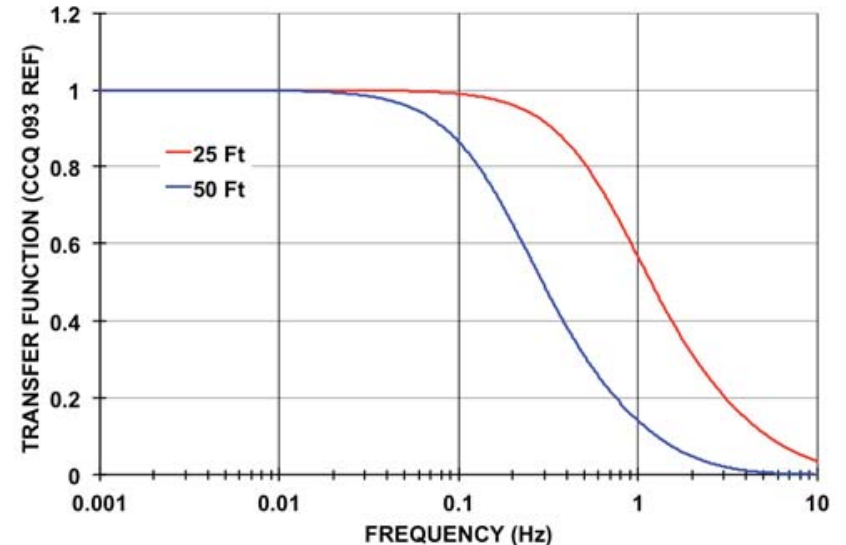

Figure 11. Estimated tubing response for Kulite reference tube.

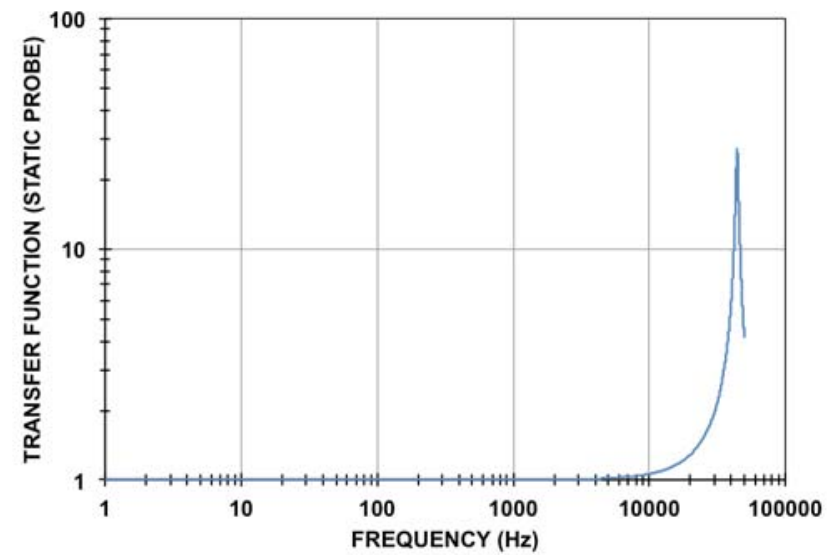

Figure 12 Estimated measurement response of the unsteady static pressure probe.

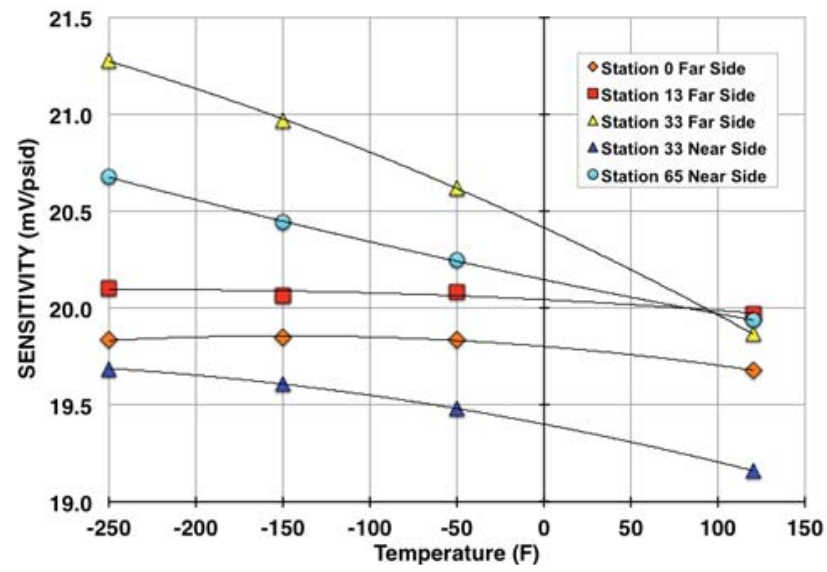

Figure 13. Examples of the temperature influence on the unsteady wall pressure transducer sensitivities. 


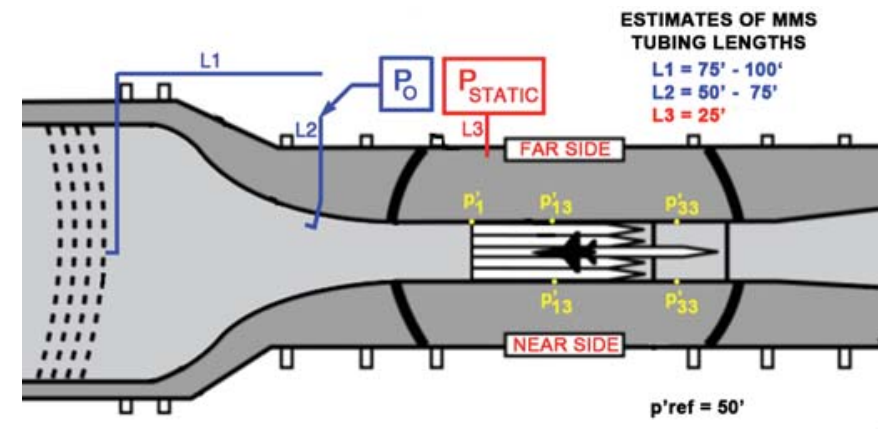

Figure 14. Sketch of tubing layout for NTFs Mach Measurement System and unsteady pressure system.

It is recognized that the mean or averaged pressure for both the total and static pressure is desired, but the repeatability of these measurements can be influenced by low frequency variations over the averaged period. This is illustrated in the time varying deviation of the Mach and pressure measurements shown in Figure 15. The dashed horizontal lines represent the NTF acceptable variation in Mach number compared to the desired Mach number. These variations are an order of magnitude better than the proposed flow quality requirements of AGARD ${ }^{18}$.

The spectra of the DAS data shown in Figure 16 indicate that the MMS total and static pressures are approximately $157^{\circ}$ out of phase at low frequencies (i.e., $0.033 \mathrm{~Hz}$ corresponding with a 30 second period). This difference can possibly be linked to the plenum resonance and or the low frequency damping effect of the Kulite and or the MMS reference system.
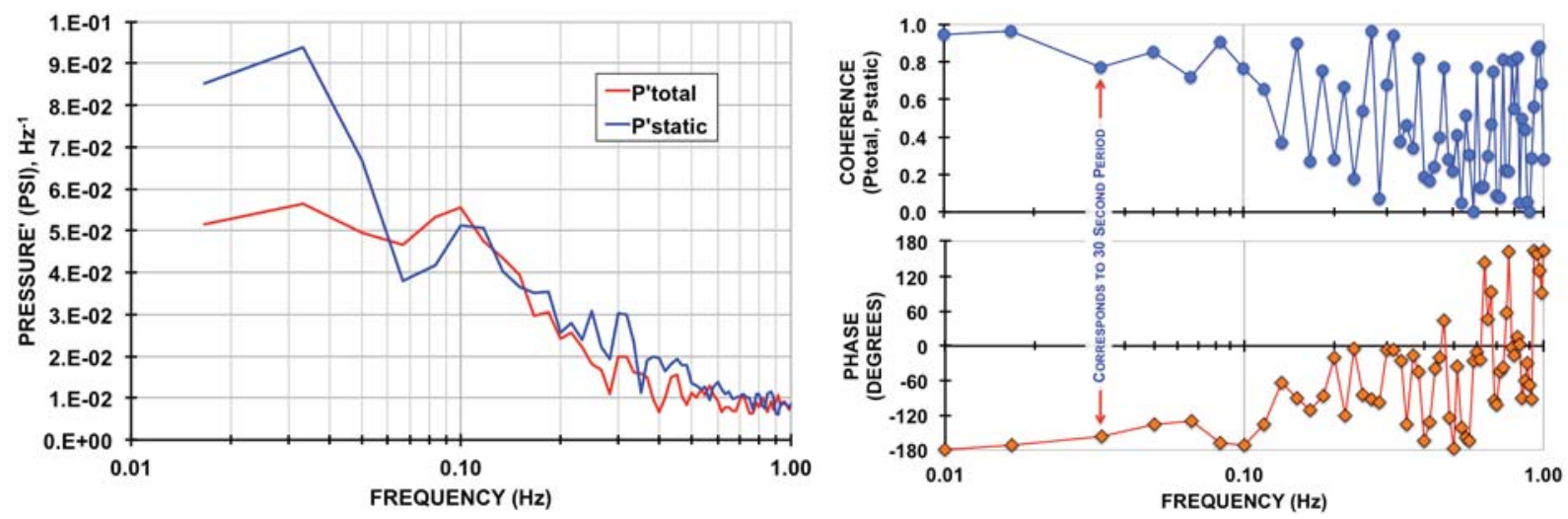

Figure 16. Power spectra, coherence, and phase relationship of Fluke total and static pressure system, Mach $=0.8500, \mathrm{P}_{\mathrm{O}}=30.12 \mathrm{psi}, \mathrm{T}_{\mathrm{O}}=-49.90^{\circ} \mathrm{F}, 120$ second period, unchoked configuration, $k=8$.

The time varying pressures are combined to achieve time varying Mach number and any phase mismatch can bias the result. The static pressure is $180^{\circ}$ out of phase with the Mach number while the total pressure is only $18.5^{\circ}$ out of phase with Mach number at $0.033 \mathrm{~Hz}$ as shown in Figure 17.

In general, it is assumed that the pressure measurements acquired by the NTF are not influenced by any resonance issues associated with the Fluke transducers or related tubing. The description shown below is intended to support that assumption. The analysis of the response of the MMS will focus on two aspects that influence the magnitude and phase shift of the measurement: the electronic response of the Fluke system and the response of the tubing used to interface the pressure device to the measurement locatation. 
Tubing length estimates were made for the MMS total pressure and static pressure measurements using the procedures described above for the Kulite transducers. There are two choices of total and static pressure locations and the preliminary tubing lengths estimates are shown in Figure 14. An example of the corresponding tubing response is shown in Figure 18 for different static pressures. Even though the tubing resonance amplitude grows with increasing tunnel pressure, there is a mimimal influence below $1 \mathrm{~Hz}$ where the data is digitally filtered.
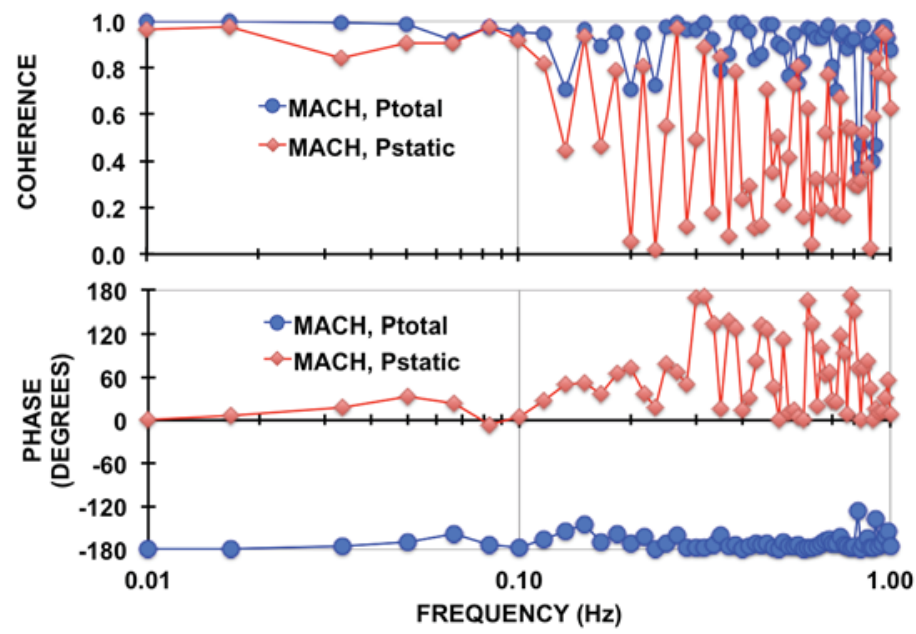

Figure 17. Coherence and phase relationship of Fluke total and static pressure to Mach number,

Mach $=0.8500$, Po $=30.12 \mathrm{psi}$, To $=-49.90^{\circ} \mathrm{F}, k=8$.

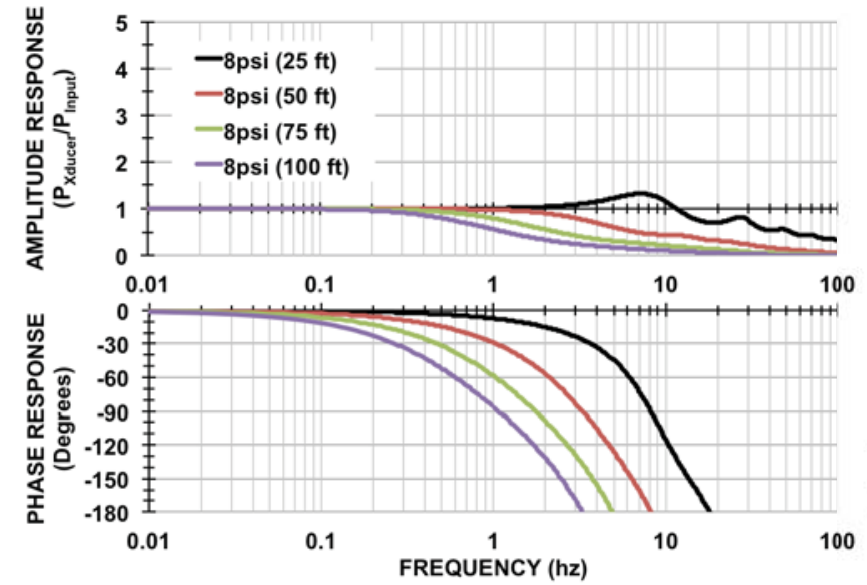

(a) $P_{S}=8$ psia
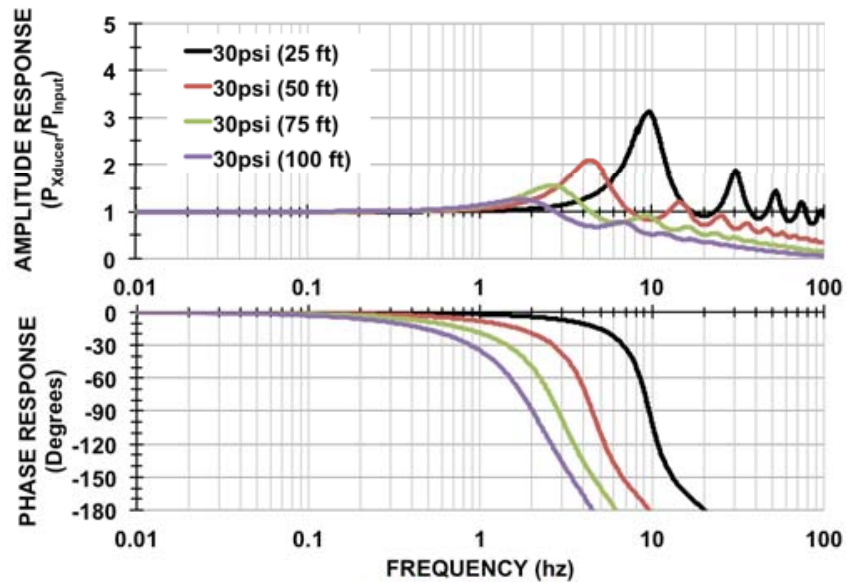

(c) $P_{S}=30$ psia
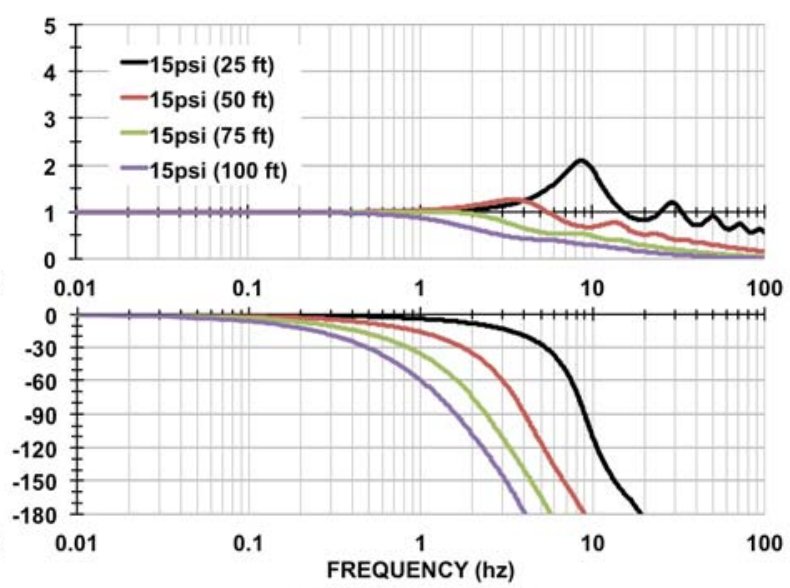

(b) $P_{\mathrm{S}}=15$ psia
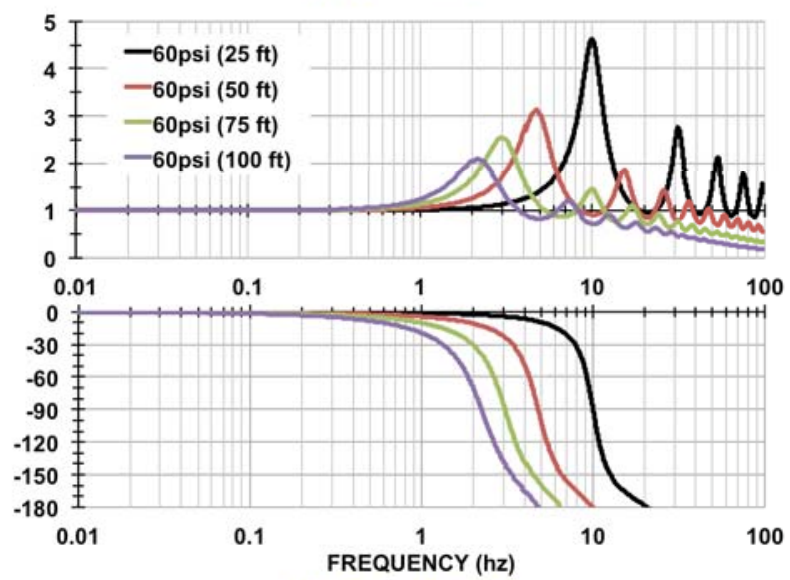

(d) $P_{S}=60$ psia

Figure 18. Theoretical transfer function (gain) and phase of MMS tubing system for various static pressure conditions, $\mathrm{To}=\mathbf{7 2}^{\circ} \mathrm{F}$. 


\section{Unsteady Pressure Characteristics for Different Model Configurations}

\section{A. Empty Tunnel (Centerline Pipe)}

The primary purpose of the centerline probe test was to calibrate the NTF test section Mach number while obtaining an empty tunnel wall signature over the full range of tunnel operation. The test section Mach number distribution was determined as a function of total pressure, temperature, and Mach number. During various portions of the testing, experimentation was conducted with vortex generators and the configuration of the second throat. The effectiveness of these devices focused on the impact to the low frequency unsteady flow field, which related directly to data quality. The unsteady characterization of the empty tunnel was a secondary effort that did not interfere with the primary objective of calibration.

This test consisted of cryogenic testing phases at $-250,-150$, and $-50 \mathrm{deg} F$; along with air mode testing at $120 \mathrm{deg} F$. This range of temperatures covered the full envelope of conditions that are typically tested at the NTF. Since test section unsteady transducers

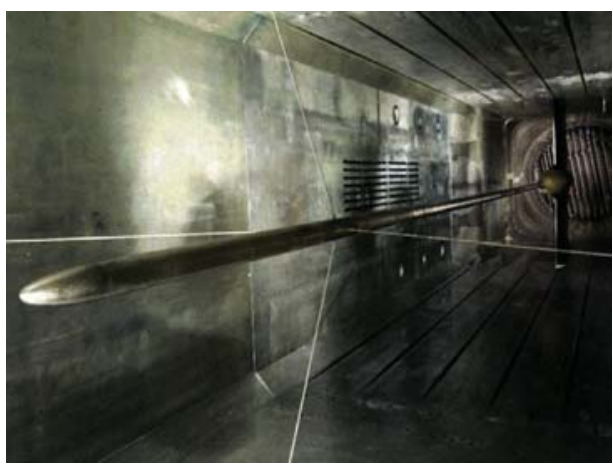

Figure 19. Photo of the centerline pipe in the NTF test section. were not available to evaluate the second throat configuration, this section of the paper will only highlight the impact of the VGs. Data acquisition was completed on 15 second intervals with a DAS scan rate of $50 \mathrm{~Hz}$ and a DDAS rate of $12800 \mathrm{~Hz}$.

The centerline probe shown in Figure 19 is a long cylindrical pipe with a diameter of three inches that extended 32 feet longitudinally. The fore (upstream) end of the probe converges to a rounded point and extends 8.5 feet into the contraction of the tunnel. Four cables that interface with the probe at tunnel station -4.395 feet supported the forward section of the probe. Each cable exits the tunnel wall at station -6.763 feet, which equates to a forward sweep angle of 30 degree. The probe has a total of 320 orifices that are arranged in four longitudinal rows that are situated circumferentially at 90 degree intervals. The primary row of orifices extends the length of the pipe at a nominal spacing of 3.0 inches with 1.0 inch spacing near test section station 0 feet and for stations 9 feet to 17 feet.

An example of the test section centerline Mach number distribution is highlighted in Figure 20a. These data are used for wall-induced-blockage corrections ${ }^{19}$. The impact of VGs and the second throat on the sidewall Mach number distribution is shown in an example highlighted in Figure 20b. The unsteady pressure transducers used for this phase of testing were limited to the 33 foot station for both far sidewall and near sidewall locations. These unsteady pressure transducers are located four feet upstream of the VGs and seven feet downstream of the second throat. This location is conducive for evaluating the VGs but not the effectiveness of the second throat.

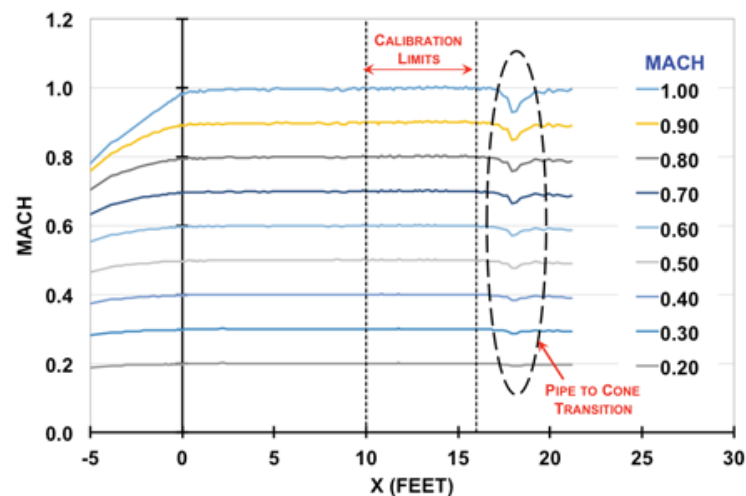

(a) CENTERLINE PIPE

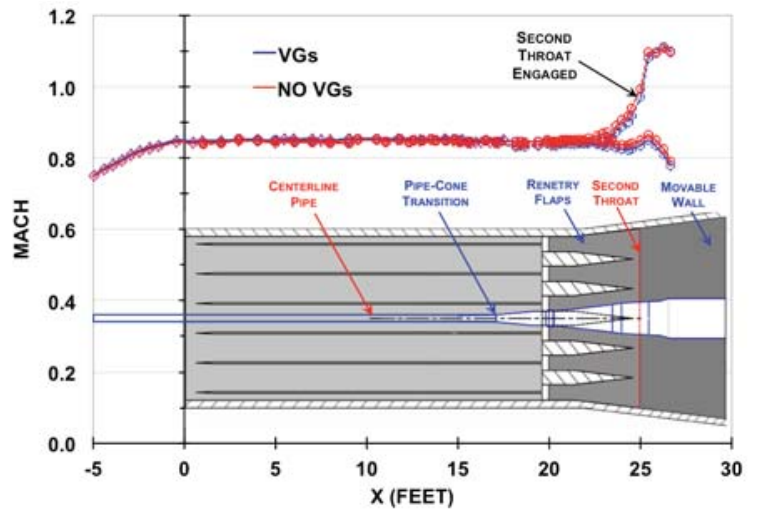

(b) SIDEWALL

Figure 20. Mach distribution $P o=20 \mathrm{psi}, \mathrm{To}=120^{\circ} \mathrm{F}$.

To gain an understanding of the sources related to the spectra, the frequency was normalized using the following relationship:

$$
\text { Non-dimensional frequency }=(\text { frequency })(\text { Length }) /(\text { Velocity })
$$


where the velocity is based on local conditions or the speed of sound. Coherent structures related to vorticity follow streamlines associated with local velocity conditions and typically will be correlated with Mach number. Similarly coherent structures related to sound fields are associated with the speed of sound and may also be correlated to Mach number. However, coherent structures that are not correlated with Mach number, such as machinery that operates at a constant condition, will be assumed to be sound related.

The benefit of the VGs on the unsteady pressure field for the empty tunnel configuration is highlighted in Figures 21 through 24. The data shown in Figure 21 was integrated over two ranges to highlight the contribution of the high energy low frequency unsteadiness to the overall fluctuating pressure. While there is a benefit of VGs over the entire bandwidth, the integration from 0 to $10 \mathrm{~Hz}$ reveals that the stabilizing low frequency benefit of the VGs are for Mach numbers greater than 0.6. This will be confirmed in the following spectral analysis.

A general observation of the influence of the VGs on the unsteady pressure field is that the VGs provide an overall stabilization and reduction of the magnitude of the broadband pressure fluctuations as seen in Figure 23. Two correlated coherent peaks are also identified at non-dimensional frequencies of 19 and 45.4 for both the baseline and VG configurations. The wavelength associated with the non-dimensional frequency of 19 is 0.63 feet. This is consistent with the nearby re-entry flap geometry or sidewall boundary layer scales. The peak that occurs at the non-dimensional frequency of 45.4 is consistent with vortex shedding from the guy wires that support the centerline pipe. It should also be noted that the frequencies associated with the fan blade and fan blade interactions with the inlet and exit guide vanes are not present in this data.

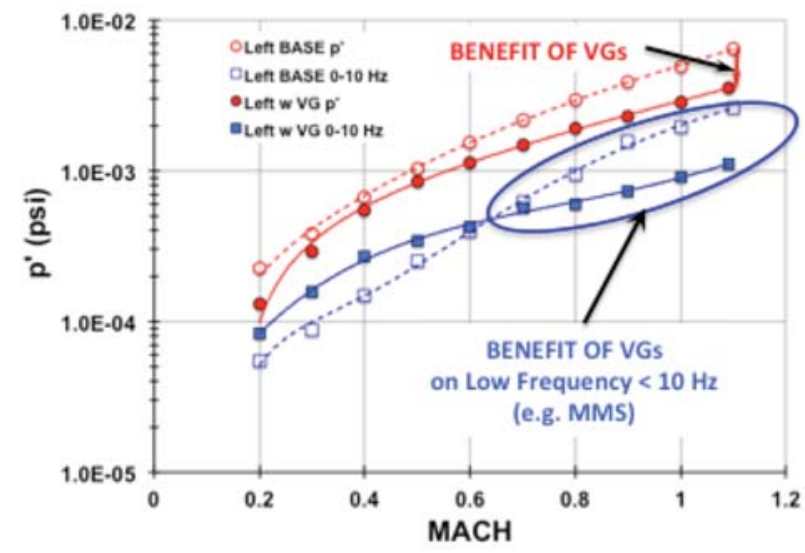

Figure 21. Influence of VGs on the pressure fluctuations measured at the model arc sector, $P_{O}=20 \mathrm{psi}, \mathrm{T}_{\mathrm{O}}=120^{\circ} \mathrm{F}$.

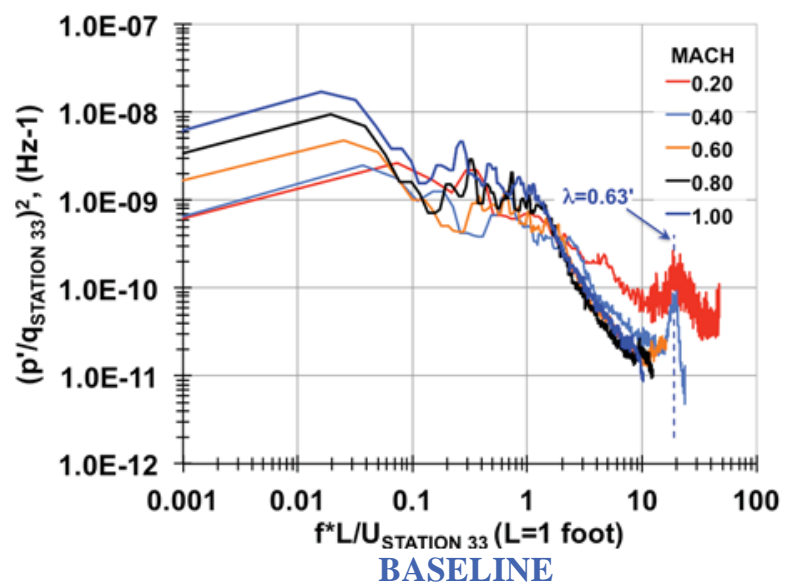

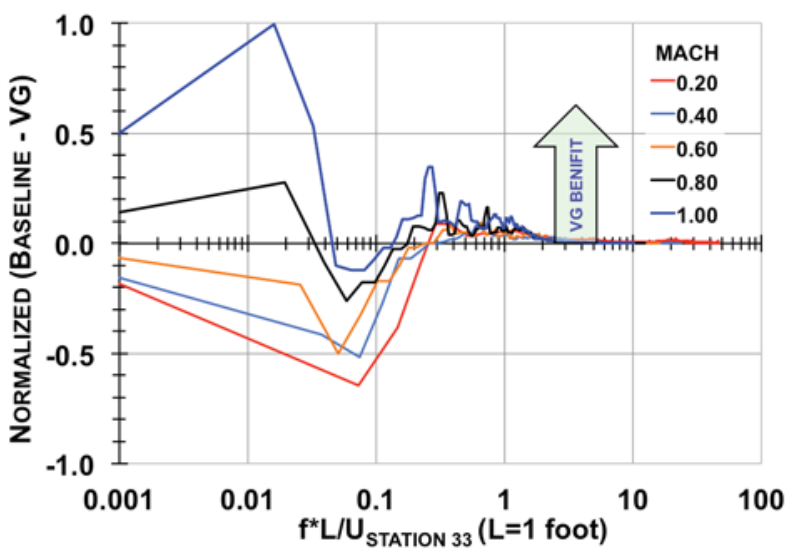

Figure 22. Difference between baseline and VG configuration on the pressure fluctuations measured at the model arc sector, $P_{O}=20 \mathrm{psi}, \mathrm{T}_{\mathrm{O}}=120^{\circ} \mathrm{F}$.

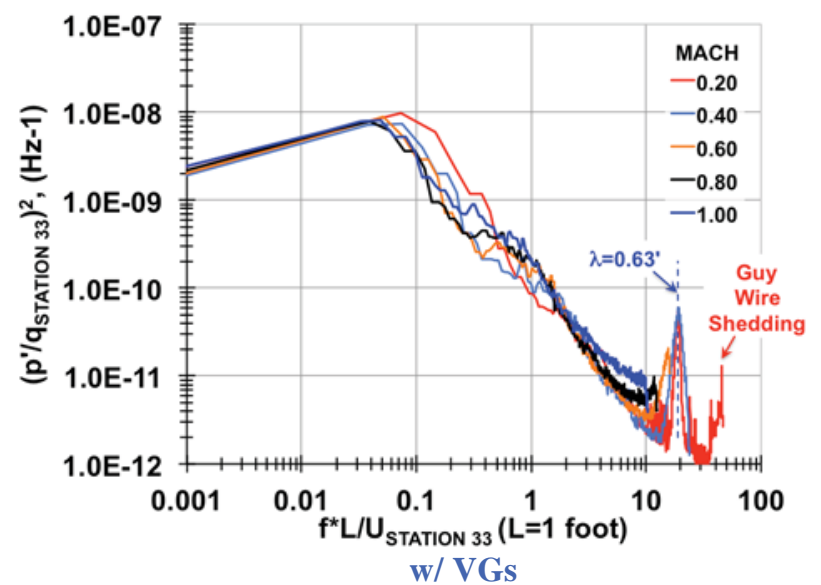

Figure 23. Influence of VGs on the pressure spectra measured at the model arc sector, reference local velocity. $P_{O}=20 \mathrm{psi}, \mathrm{T}_{\mathrm{O}}=120^{\circ} \mathrm{F}, k=240$. 
Subtracting the baseline spectra from the VG configuration spectra results in data that highlights the range of frequencies where the VGs are beneficial as seen in Figure 22. Comparing the baseline spectra with the spectra from a VG configuration in Figure 23, one can identify apparent coherent features that occur between nondimensional frequencies between 0.2 and 1.0 in the baseline configuration but are not present in the VG configuration and do not appear to be correlated with Mach number. Re-evaluating this same data with a fixed reference velocity using the speed of sound is shown in Figure 24. The coherent structure identified in the baseline configuration has a fixed wavelength of 6.89 feet and does not vary with Mach number. Similarly a fixed wave length of 5.41 feet can be identified in the VG configuration. It is not clear as to the source of these disturbances and will require further investigation.
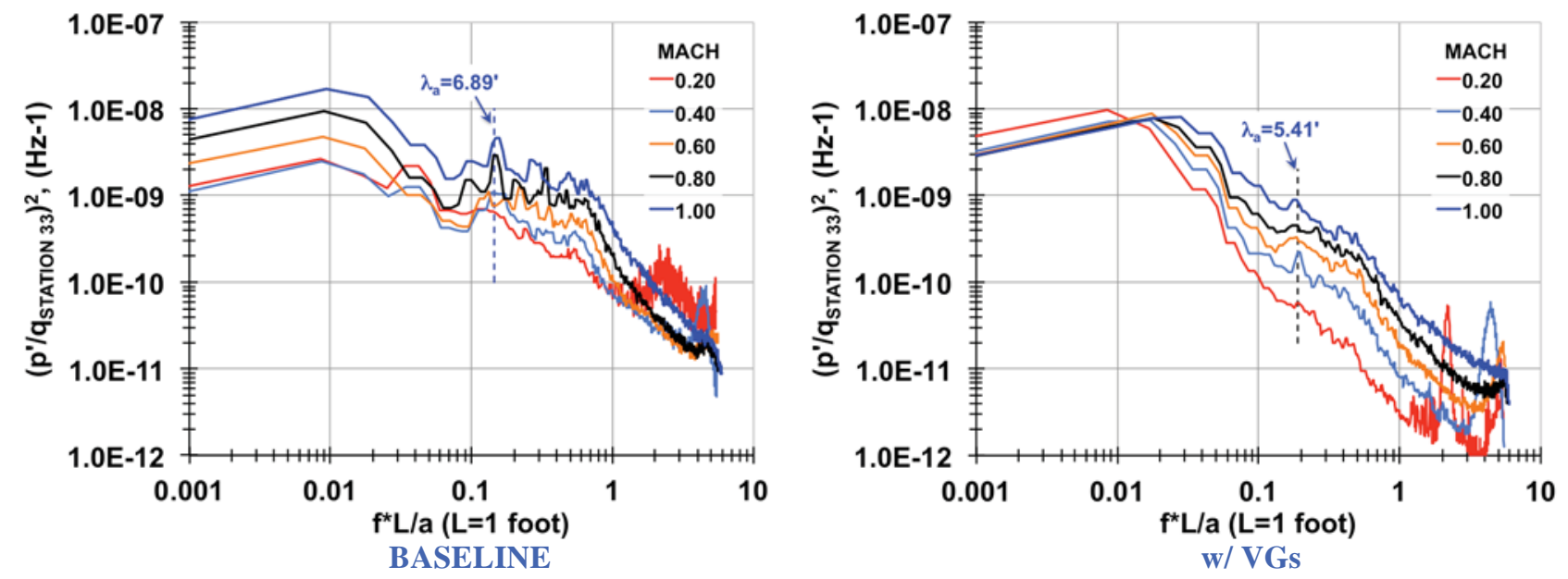

Figure 24. Influence of VGs on the pressure spectra measured at the model arc sector, reference speed of sound, Po $=20$ psi, $\mathrm{To}=120^{\circ} \mathrm{F}, k=240$.

\section{B. Empty Tunnel (Rake)}

The 7-foot Flow Survey Rake shown in Figure 25 was used to characterize the mean temperatures, mean pressures, and fluctuating pressures across the NTF test envelope. The layout and type of probes is shown in Figure 26. Crosssectional temperature and pressure surveys of the flow were generated as the rake was rotated $360^{\circ}$ in the test section. The results of the mean temperature and pressure surveys are outside the scope of this paper.

Spacing between the probes was four inches across the leading edge of the rake and the probe measurements covered an 80 inch diameter circular cross-section of the 8.2 foot test section. Seven unsteady surface mounted pressure probes were mounted on the test section and diffuser walls to be correlated with the free stream unsteady pressure measurements. Data were taken in air mode at $120^{\circ} \mathrm{F}$ and with cryogenic nitrogen at $-50^{\circ} \mathrm{F}$ and $-250^{\circ} \mathrm{F}$. Mach numbers ranged between 0.1 and 0.95 with total pressures between 16

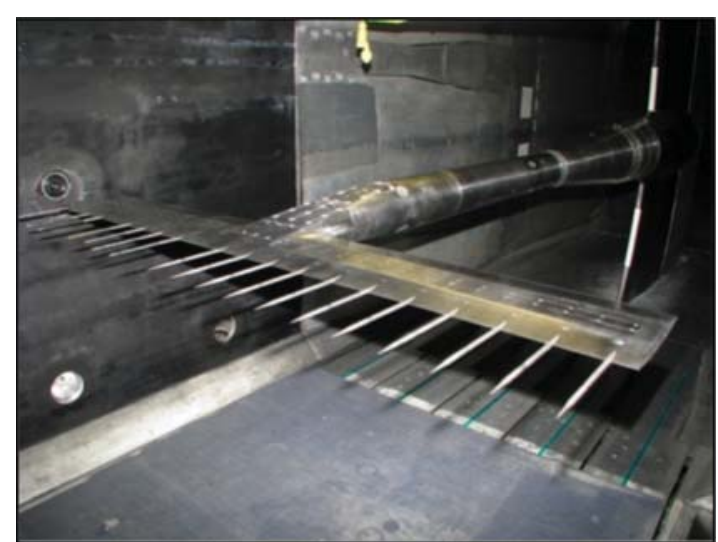

Figure 25. Photograph of the 7-foot survey rake shown at the $0^{\circ}$ roll position. psi and 120 psi across all three test temperatures. This paper will focus on the transonic conditions, particularly the second throat choked condition at a Mach number of 0.85 .

Upon reaching the desired flow condition, roll polars were conducted with the rake positioned in the center of the test section at $0^{\circ}$ angle-of-attack. A typical run is referenced to a roll angle of $90^{\circ}$ (Rake vertical with probe 1 near the ceiling) and moved between $+/-180^{\circ}$ to $-/+180^{\circ}$ in $15^{\circ}$ roll increments, pausing 10 seconds to collect data at each point. The NTF's DDAS acquired the fluctuating pressure signals from the rake and walls at frequencies between $40.96 \mathrm{kHz}$ and $102.4 \mathrm{kHz}$. 
The influence of the second throat on the Mach number distribution is shown in Figure 27. The baseline wall setting begins to decelerate flow in the diffuser past the reentry flap region located at the 25 foot station. A minor increase in the Mach number is present at the second throat hinge line. At no time did the flow spike above Mach 1 at the second throat for the baseline wall settings. When the second throat was engaged the flow began to accelerate between station 20 and 25 . The flow reaches its peak speed

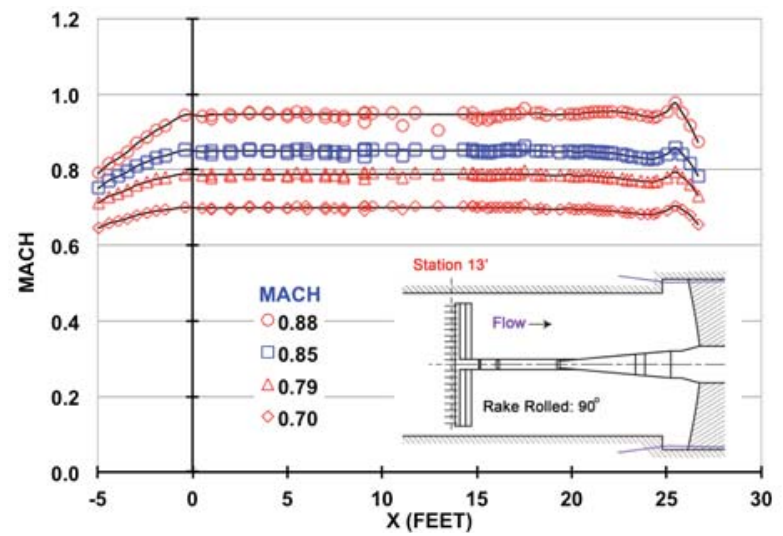

(a) BASELINE

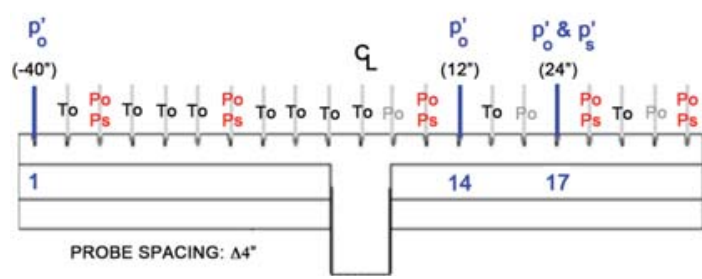

Figure 26. Schematic of the layout of the NTF 7-foot Survey Rake.

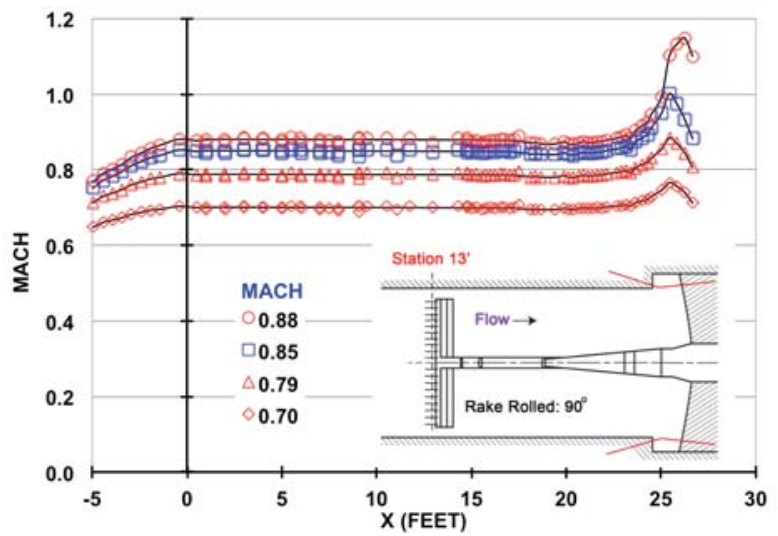

(b) CHOKED

Figure 27. Sidewall Mach distribution for unchoked (baseline) and choked configurations, $\mathrm{To}^{\mathrm{o}}=121^{\circ} \mathrm{F}$, $20<$ Po (psia) $<35$.

at the second throat located at station 25 feet, becoming supersonic for free stream Mach numbers near 0.85 . The second throat Mach number reaches 1.14 for a test section Mach of 0.88 . The flow begins a sharp deceleration downstream of the shock as the flow enters the diffuser.

An example of the pressure fluctuation uniformity in the test section is shown in Figure 28 for a Mach number of 0.85 and second throat choked condition. The lack of variations in the magnitude of the fluctuating data is consistent with the power spectra for different roll positions that are shown in Figure 29. The fluctuating pressure across the test section contains most of the broadband energy below a non-dimensional frequency of $2(1.58 \mathrm{kHz})$. There are two coherent structures that exist above non-dimensional frequencies of 2 for this condition, i.e., non-dimensional frequencies of 4.75 and 15.2 and corresponding to $3740 \mathrm{~Hz}$ and $12 \mathrm{kHz}$, respectively. The sources for the high frequency sources are not identified but are not correlated to the fan or fan interactions. The low frequency data

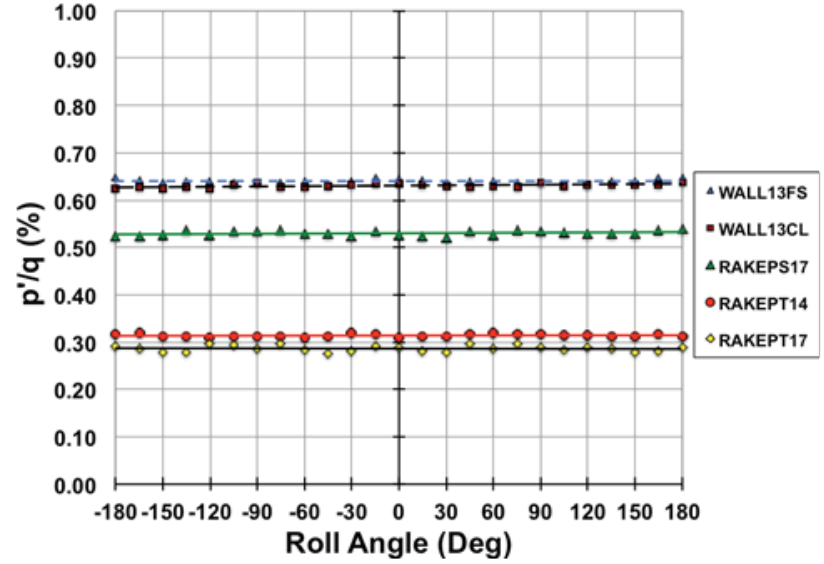

Figure 28. Pressure fluctuations for choked configuration, $M=0.8501, R e / f t=49.4 \times 10^{6}$, To $=-251.1^{\circ} \mathrm{F}, \mathrm{Po}=44$ psia.

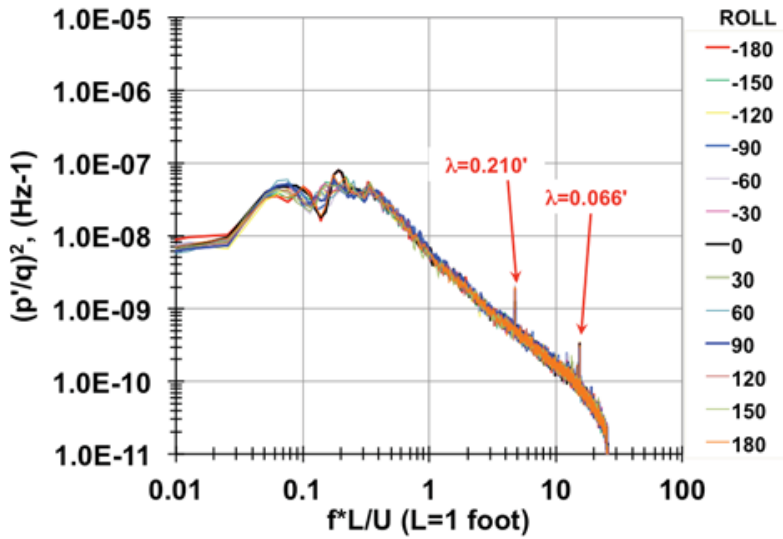

Figure 29. Roll sweep for choked configuration, p'17 STATIC $_{\text {, }} \mathrm{M}=0.8503, \mathrm{Re} / \mathrm{ft}=14.7 \times 10^{6}, \mathrm{To}=-51.6^{\circ} \mathrm{F}$, Po $=35$ psia, $q=11.05$ psi, $k=240$. 
characterized in Figures 30 and 31 are related to the rake interaction with the sidewalls and or edge tone noise generated by the slots. These data are representative of other Mach and Reynolds numbers as shown in Figure 32.

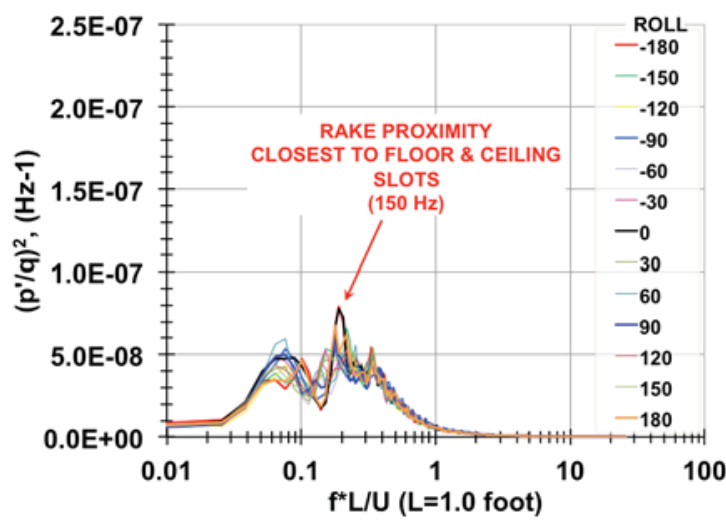

Figure 30. Free Stream pressure fluctuations (p'17 $7_{\text {STATIC }}$ ) for choked configuration, $\mathrm{M}=\mathbf{0 . 8 5 0 3}$, To $=-51.6^{\circ} \mathrm{F}$, Po $=35 \mathrm{psia}, \mathrm{Re} / \mathrm{ft}=14.7 \times 10^{6}$, $\mathrm{q}=\mathbf{1 1 . 0 5} \mathrm{psi}, k=\mathbf{2 4 0}$.

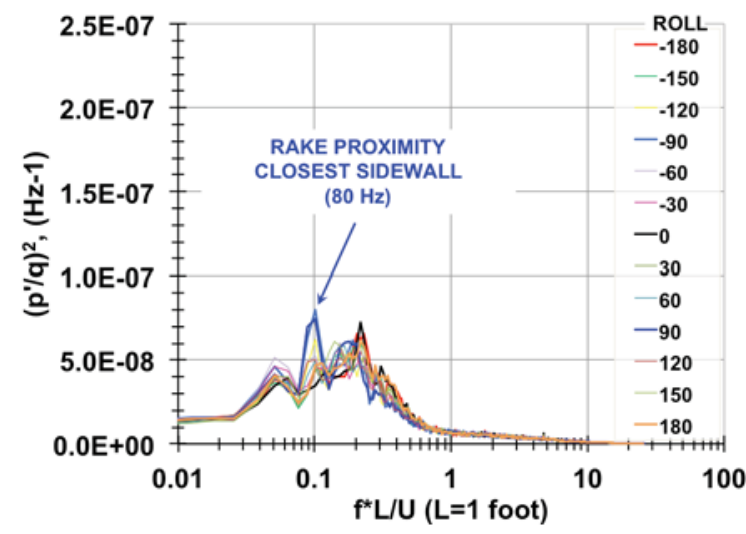

Figure 31. Near sidewall fluctuations (p'13 ${ }_{\text {STATIC }}$ )

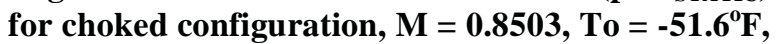
$\mathrm{Po}=35 \mathrm{psia}, \mathrm{Re} / \mathrm{ft}=14.7 \times 10^{6}, \mathrm{q}=11.05 \mathrm{psi}$, $k=240$.

The effectiveness of the second throat for a Mach number of 0.85 is illustrated in Figure 33. The choked condition not only stabilizes the low frequency stability of the high-speed diffuser $(<10 \mathrm{~Hz})$ but also reduces the rake interactions with the tunnel $(10<\mathrm{Hz}<1000)$. The normalized pressure fluctuations $(\mathrm{p} / \mathrm{q})^{2}$ is reduced by a factor of 5. This is particularly important to the stability of Mach number and dynamic pressure repeatability, which has a large influence on repeatability of model lift and drag performance data. ${ }^{20}$ The benefit of the second throat on the unsteady pressure field is not realized for frequencies above $1000 \mathrm{~Hz}$.

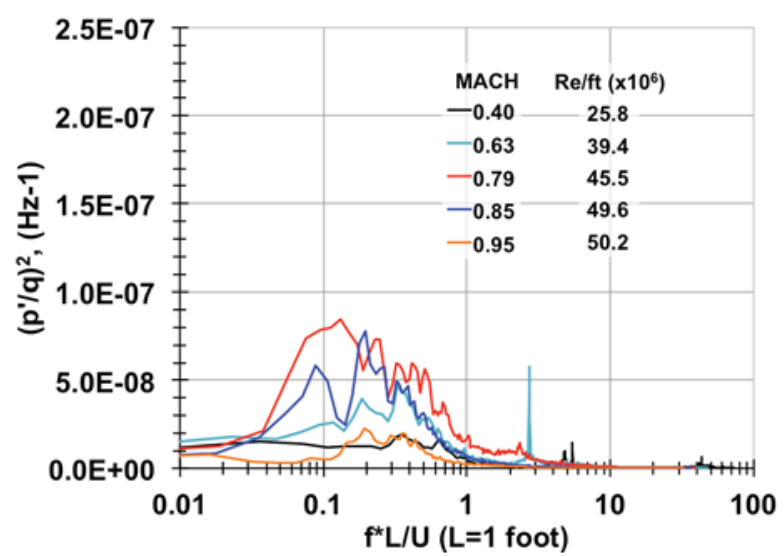

Figure 32. Free Stream fluctuations (p'17 STATIC $)$ for Choked configuration, To $=-251.6^{\circ} \mathrm{F}, \mathrm{Po}=42$ psia, Roll angle $=0^{\circ}, k=240$.

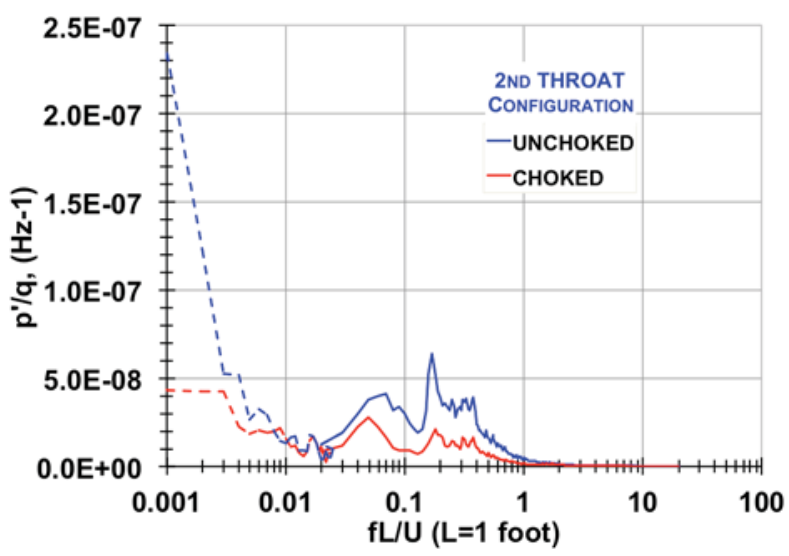

Figure 33. Comparison of free stream fluctuations

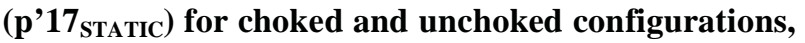
$\mathrm{M}=0.85$, To $=120^{\circ} \mathrm{F}, \mathrm{Po}=30 \mathrm{psia}, \mathrm{q}=9.5 \mathrm{psi}$, Roll angle $=0^{\circ}$. 


\section{Common Research Model}

The Common Research Model (CRM) shown in Figure 34 is a full-span, $2.7 \%$ scale model of a state-of-the-art commercial aircraft. The critical geometric components of this model are its wingspan of 62.47 inches, fuselage length of 66.73 inches, and the mean aerodynamic chord of 7.45 inches as shown in Figures 35. The force and moment data associated with the CRM is outside the scope of this paper and is described in Reference 21.

The effectiveness of the second throat is dependent on the shock strength formed at the minimum area downstream of the test section. The second throat shock strength was examined with the sidewall pressure distributions measured along the centerlines of the tunnel sidewalls (rows 9 and 19) shown in Figure 36. The baseline Mach distribution for a 0.85 Mach

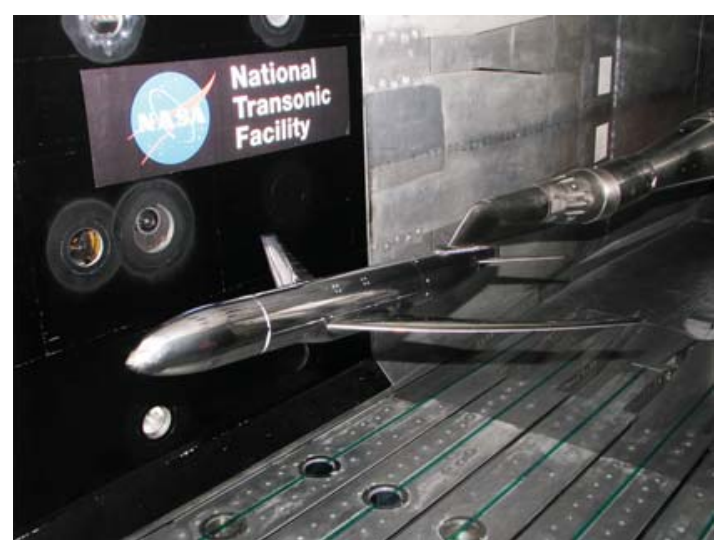

Figure 34. Photograph of the Common Research Model.

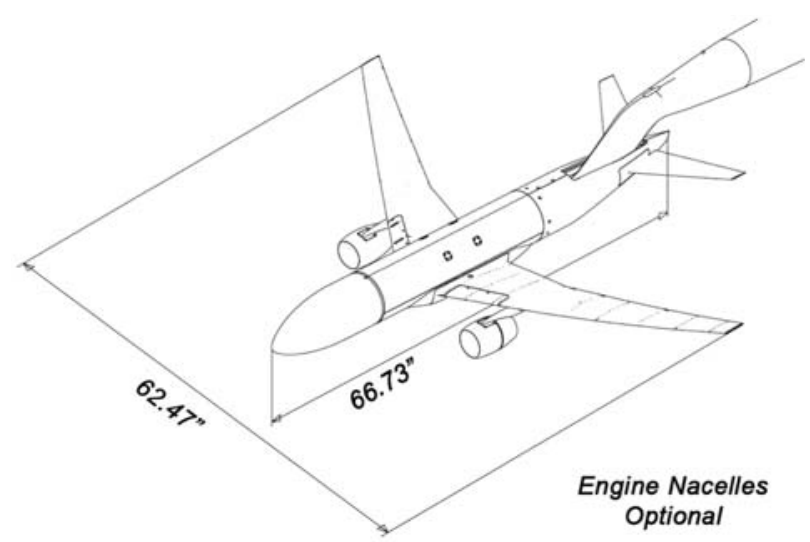

Figure 35. Sketch of the Common Research Model.

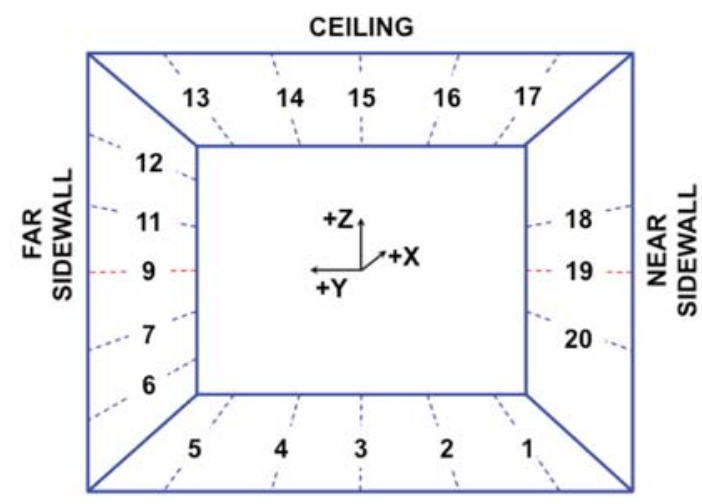

FLOOR

Figure 36. NTF geometry for sidewall rows of pressure orifices.

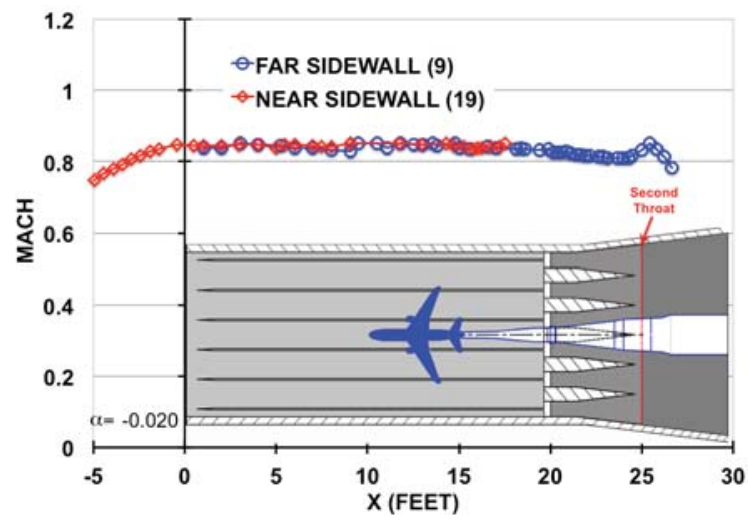

(a) BASELINE

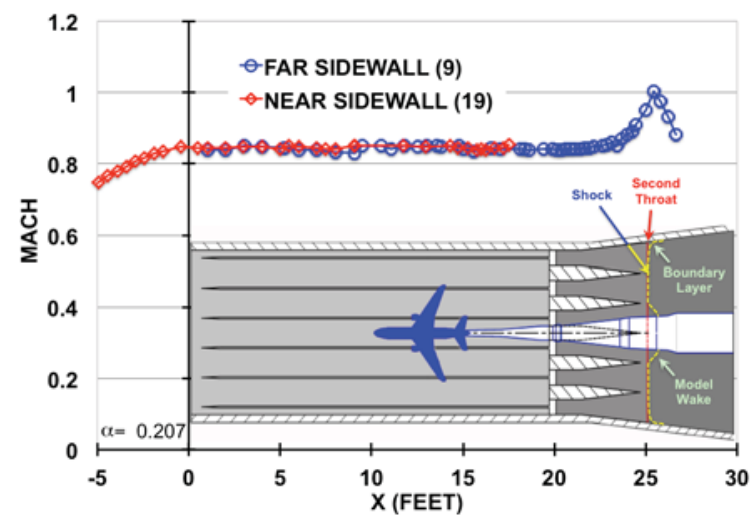

(b) CHOKED

Figure 37. Example of test section Mach number distribution for baseline condition, $M=0.85, T 0=121^{0} \mathrm{~F}$, $\operatorname{Re}_{r}=4.99 \times 10^{6}$.

condition is shown in Figure 37a. This wall signature is uniform through the test section and does not go sonic at any stream wise location. The wall signature for the choked condition shown in Figure 37b indicates that the flow becomes sonic at the second throat located at station 25 feet.

The peak magnitude of the pressure fluctuations typically occur near a Mach number of 0.8 where compressible effects at the end of the test section begin to attenuate the upstream moving acoustic field coming from the high 
speed diffuser as shown in Figure 38 22 23. The presence of the model increases the fluctuating pressure levels to $0.67 \%$ at a Mach number of 0.85 .

Examining the spectra for a Mach number of 0.85 shown in Figure 39, one can identify a model related coherent structures at a non-dimensional frequency of $0.72(670 \mathrm{~Hz})$, $0.106(100 \mathrm{~Hz}), 0.053(50 \mathrm{~Hz})$ and $0.018(17 \mathrm{~Hz})$. Comparing Figure 39 and 40 one can identify frequencies that are common to the choked and unchoked configurations. These include non-dimensional frequencies of 0.72 and 0.018 where the magnitude of the 0.72 structure is the largest feature shown in the choked and unchoked configurations. In an attempt to identify the sources of these coherent features it was necessary to evaluate the fan and its interactions with the

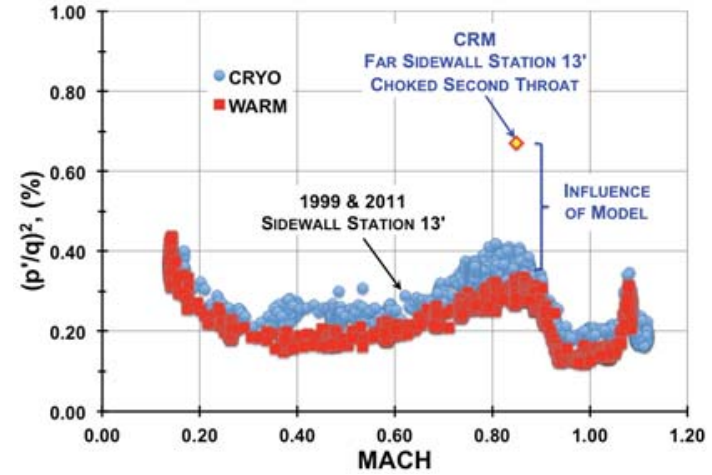

Figure 38. Sidewall pressure fluctuations. inlet and exit guide vanes. There is also a coherent structure identified at a non-dimensional frequency of 0.018 for both the choked and unchoked configurations. This coherent structure has a wavelength of approximately 55 feet. This is the approximate distance of the arc sector to the turning vanes. No other features related to model geometry (e.g., wing span, fuselage length, etc.) were identified for these wind tunnel conditions. Examining the phase relationship between 13 and 33 foot station indicates a positive phase that suggests that this coherent structure is moving downstream.

The fan interaction frequency is a combination of the number of fan blades plus the number of exit guide vanes plus the number of inlet guide vanes. The only coherent feature related to the fan is identified in Figure 40 at a nondimensional frequency of 0.72. It is recognized that this feature was not apparent in the empty tunnel configurations. Therefore it is believed to be excited by a model related structure. Examining the phase relationship of the 13 and 33 foot stations for the non-dimensional frequency of 0.72 indicates that this is a sound feature that is moving upstream. However, the confidence interval is low for this data set and should be re-evaluated with long period data that is currently not available.

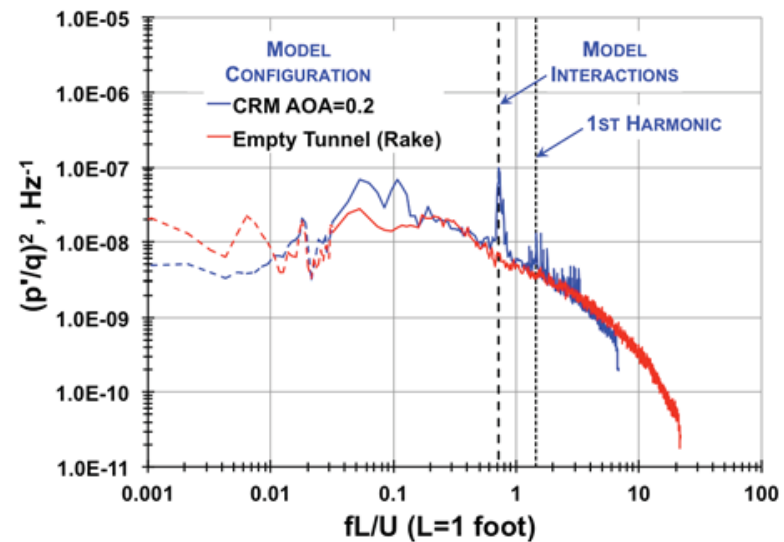

Figure 39. Comparison of far wall station 13 feet fluctuations for the CRM model and empty tunnel choke configuration. $\mathrm{M}=\mathbf{0 . 8 5}, \mathrm{q}=9.63 \mathrm{psi}$, $\operatorname{Re}_{\mathrm{C}}=4.9 \times 10^{6}, \mathrm{To}=127^{\circ} \mathrm{F}, \mathrm{T}=12$ seconds, $k=240$.

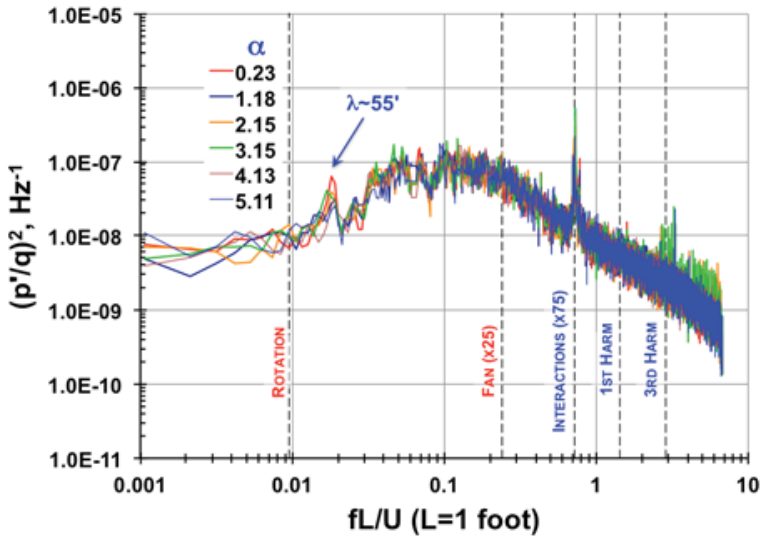

Figure 40. Normalized far wall station 13 feet spectra for $\alpha$ sweep characteristics for unchoked configuration $\mathrm{M}=\mathbf{0 . 8 4 9 5}, \mathrm{Po}=30.51 \mathrm{psi}$, $\mathrm{q}=9.6285 \mathrm{psi}, \mathrm{To}=128.71^{\circ} \mathrm{F}, \operatorname{Re}_{\mathrm{C}}=4.91 \times 10^{6}, k=24$.

To focus on the features that impact Mach stability and the impact of the model wake has on the tunnel flow field, the spectra shown in Figure 40 were reanalyzed by integrating only frequencies less than $10 \mathrm{~Hz}$. The energy contained in this bandwidth is approximately $15 \%$ of the total energy. This upper frequency limit of $10 \mathrm{~Hz}$ corresponds to a non-dimensional frequency of 0.1. As the model angle of attack is increased for the unchoked tunnel configuration, the magnitude of the pressure fluctuations increase linearly as shown in Figure 41. A study of the spectra suggests that the model wake generates strong low frequency unsteadiness for non-dimensional frequencies less than 0.01 that couple the model wake to the high-speed diffuser. Once a shock is established at the second throat, the low frequency unsteady effects subside, indicating that the shock acts as a barrier for the lower frequency energy coupling from the wake to the high speed diffuser. This stabilizing effect improves the Mach 
stability as impacted by the model angle of attack is significantly reduced by implementing a choke at the second throat.

The benefits of the VGs that were identified in the empty tunnel configurations are not realized with a model in the tunnel. Figure 42 shows that the broadband and the model coherent structures are not influenced by the addition of the VGs for the choked condition. However, the wind tunnel fan power increased with the addition of the VGs making the benefits of the VGs undesirable.

Taking advantage of NTFs capability to vary the speed of sound is seen in the correlation to the speed of sound is shown in Figure 43. As the temperature decreases the broadband normalized pressure fluctuations (p'/q) ${ }^{2}$ increases. The coherent structure collapses to a non-dimensional frequency of $0.62\left(\lambda_{\mathrm{a}}=1.61 \mathrm{feet}\right)$. There are not any model or wind tunnel dimensions that can be readily associated with this length scale. This structure was characterized as a model related feature since it was not identified in either of the empty tunnel configurations described above. However, the correlation of the non-dimensional frequency using free

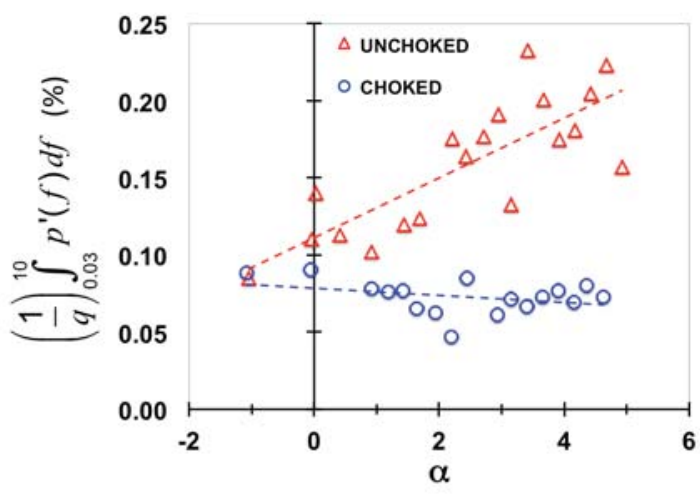

Figure 41. Benefit of second throat, data integrated from $0-10 \mathrm{~Hz}$ for $\mathrm{Mach}=\mathbf{0 . 8 4 9 5}$, Po $=30.51$ psi, $q=9.6285$ psi, To $=122^{\circ} \mathrm{F}$, $\operatorname{Re}_{\mathrm{C}}=4.98 \times 10^{6}, \alpha$ sweep. stream velocity was not as good as the correlation when using the speed of sound for different free stream Mach numbers as seen in Figure 44. Since this does not correlate with Mach number (i.e., free stream velocity), this disturbance is possibly related to an acoustic resonance that is outside the flow field.

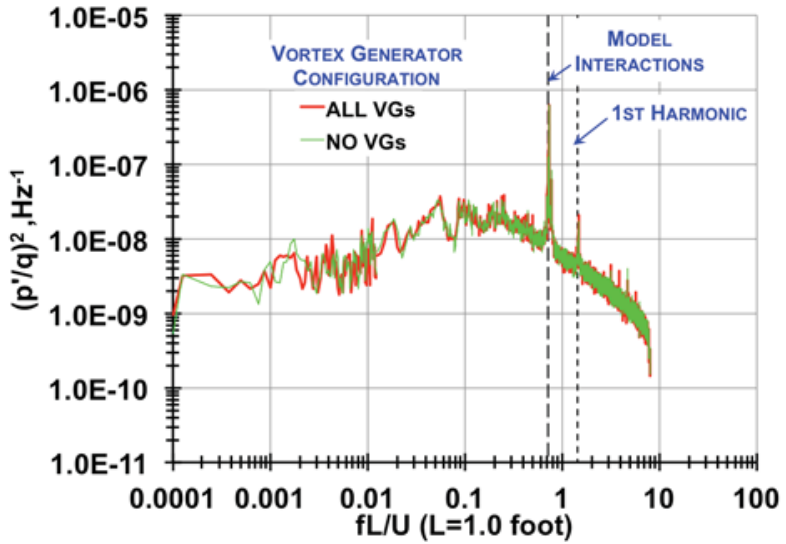

Figure 42 Influence of VGs for Mach $=0.8495$, $q=12.03, \operatorname{Re}_{\mathrm{C}}=10.0 \times 10^{6}, \mathrm{To}=-50^{\circ} \mathrm{F}, \mathrm{Po}=38.12 \mathrm{psi}$, $\mathrm{T}=60$ seconds, $k=120$, second throat choked.

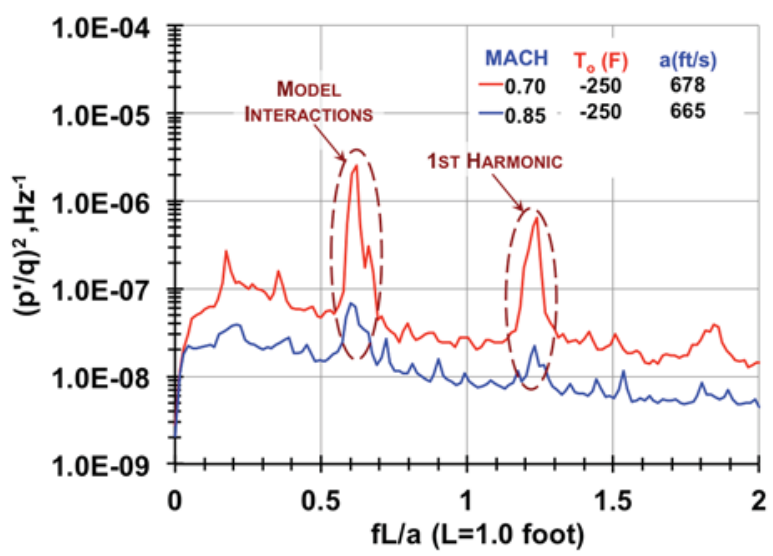

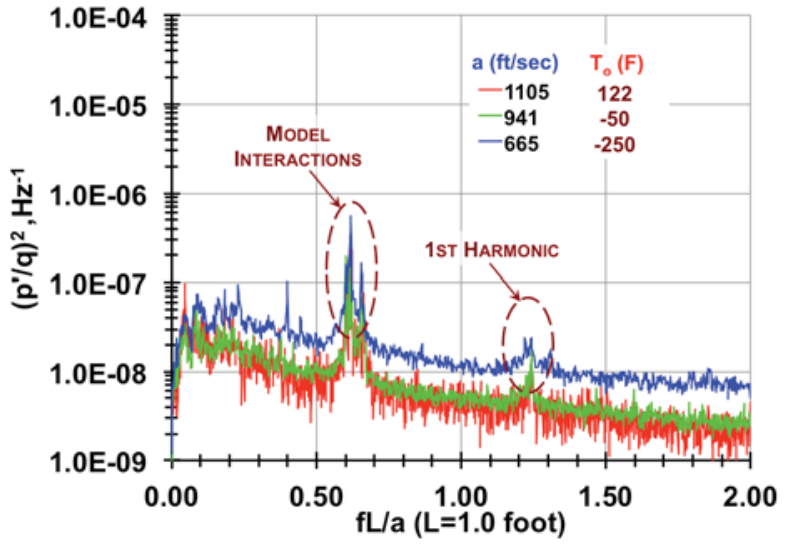

Figure 43 Correlation of the pressure fluctuation with the speed of sound (varied with temperature) for Mach = 0.85, Station 13 Far Wall, $k=120$.

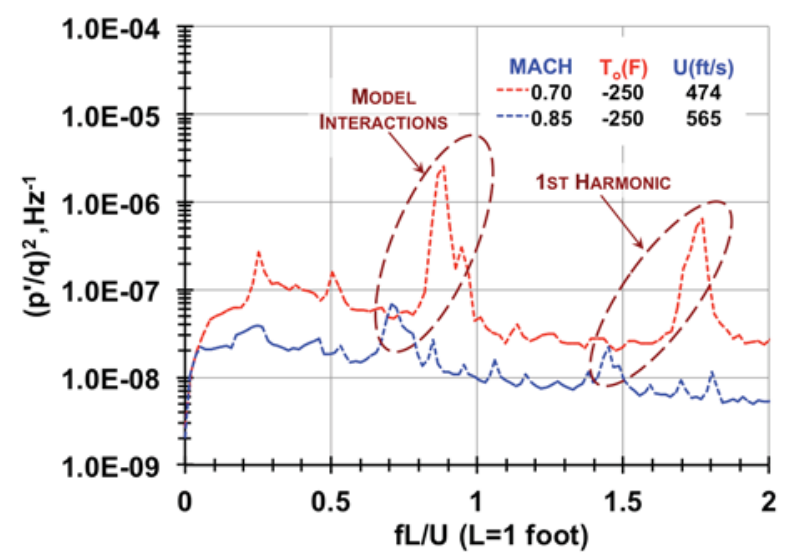

Figure 44. Model interactions for different free stream Mach numbers. 


\section{Concluding Remarks}

The focus of this paper has been on the unsteady pressure characteristics of the NTF. Three wind tunnel configurations were evaluated, two empty tunnel configurations and one configuration with a sting mounted model. Potential wind tunnel modifications that include vortex generators and a second throat were evaluated for empty tunnel and model in tunnel configurations. The unsteady pressure bandwidth of interest focused on both the low frequency, high-energy fluctuations that impact Mach stability and data repeatability, and higher frequency data that can be associated with small scale interactions of a model and the wind tunnel. The following observations highlight the impact of potential modifications investigated by this research:

- The presence of a high blockage model at transonic speeds affects the low frequency characterization of the NTF unsteady pressure field that is associated with wind tunnel turbulence. The strength of the model wake is dictated by the momentum loss associated with drag and is correlated to a very strong low frequency content that affects tunnel controls and data quality.

- The implementation of a second throat used to create a local shock was very effective in decoupling or stabilizing the wake and high-speed diffuser interactions. While the shock generated by the second throat is seen at the walls to be sonic, the core of the wake causes the flow to be subsonic. This shallow shock was very effective in reducing the low frequency model vorticity effects related to the wake. However, the subsonic wake deficit also acts as a sound conduit allowing the higher frequency upstream moving sound to move through the core of the high speed diffuser into the test section.

- Vortex generators stabilized the empty tunnel and reduced the magnitude of the fluctuations. However the VGs were not effective in the presence of the CRM model at transonic speeds. The VGs are located on the tunnel walls and do not appear to cancel the model wake effects since the wake occurs in the central core of the flow.

- The rake provided unsteady pressure data that was uniform across the test section.

As part of the NTF Facility Improvements and Data Optimization (FIDO) project, the facility is working to enable the use of the existing second throat for all transonic tunnel conditions and different types of models. While the initial results associated with the second throat looks promising, further experiments and investigations are needed to fully understand and optimize the benefits and drawbacks of using the existing second throat capability. Further investigations are also needed to characterize the potential benefits of the VGs for low Mach numbers that are influenced by the wakes of models.

\section{Acknowledgements}

The NASA Langley Research Center center director Steve Jurczyk and the NTF Subsonic Transonic Applied Refinements By Using Key Strategies (STARBUKS) and the Facility Improvements and Data Optimization (FIDO) projects under the NASA Aeronautics Test Program have supported this research. Special thanks to the NTF staff led by Roman Paryz and Eric Walker for their dedication and attention to details that made this effort successful.

\section{Disclaimer of Endorsement}

Neither the U.S. Government nor NASA endorse or recommend any commercial products, processes, or services. Reference to or appearance of any specific commercial products, processes, or services by trade name, trademark, manufacturer, or otherwise, in NASA materials does not constitute or imply its endorsement, recommendation, or favoring by the U.S. Government or NASA and are presented for reporting purposes only. The views and opinions of the author(s) expressed in this report do not necessarily state or reflect those of the U.S. Government or NASA, and they may not be used for advertising or product endorsement purposes. 


\section{References}

${ }^{1}$ Igoe, W.B., "Analysis of Fluctuating Static Pressure Measurements in the National Transonic Facility”, NASA Technical Paper 3475, (1996).

${ }^{2}$ Jones, G.S., "NTF Flow Quality using a 10 degree Cone, free stream hot-wire, and free stream fluctuating pressure probe", oral presentation, April, 15, 1996.

${ }^{3}$ Owen, F.K., Owen, A.K., "Measurement and assessment of wind tunnel flow quality", Progress in Aerospace Sciences 44 (2008) 315-348.

${ }^{4}$ Wlezien, R. White, R., Eppink, J, Liu, S., and Krause, J., "NTF Flow Quality Characterization for Laminar Flow Testing", Tufts University, September 2009.

${ }^{5}$ King, R., Andino, M., Melton, L., Eppink, J., Kegerise, M., Tsoi, A,. "Flow Disturbance Characterization Measurements in the National Transonic Facility (Invited)," AIAA 2012-0104.

${ }^{6}$ Description of a National Wind Tunnel Complex, NASA CR-198491, June 1996.

${ }^{7}$ Kovasznay, L.S.G., and Tormarck, S.I.A., "Heat Loss of Hot Wires in Supersonic Flow," Bumblebee Rep. No 127, Dept. Aero., The Johns Hopkins Univ., April 1950.

${ }^{8}$ Stainback, P.C., Johnson, C.B., and Saneett, C.B., "Preliminary Measurements of Velocity, Density, and Total Temperature Fluctuations in Compressible Subsonic Flow," AIAA 21 ${ }^{\text {st }}$ Aerospace Sciences Meeting, January 1983.

9 Stainback, P.C., "Some Influences of Approximate Values for Velocity, Density, and Total Temperature Sensitivities on Hot-Wire Anemometer Results," AIAA 24 ${ }^{\text {th }}$ Aerospace Sciences Meeting, January 1986.

${ }^{10}$ Morkovin, M.V. "Fluctuations and Hot-Wire Anemometry in Compressible Flows," AGARD-ograph 24, 1956.

${ }^{11}$ Kovasznay, L.S.G., "Turbulence in Supersonic Flow.” Journal of Aeronautical Sciences, vol. 20, no. 20, October 1953.

12 Jones, G.S., "The Measurement of Wind Tunnel Flow Quality at Transonic Speeds," Ph.D. Dissertation, Department of Engineering Science and Mechanics, Virginia Polytechnic Institute and State University, April 1991.

${ }^{13}$ Wahls, R.A., "The National Transonic Facility: A Research Retrospective (Invited)," AIAA Paper 2001-16587, January 2001.

${ }^{14}$ Hardin, J. C., "Introduction to Time Series Analysis”, NASA 1145, 1986.

${ }^{15}$ Hurst, A.M., Olsen, T.R., Goodman, S, VanDeWeert, J, Shang, T., An Experimental Frequency Response Characterization of MEMS Piezoresistive Pressure Transducers," Turbine Technical Conference and Exposition, GT2014-27159, June 2014.

16 Johansen, E. S., "Development of a Fast-Response Multi-Hole Probe for Unsteady and Turbulent Flowfields," Ph.D. Dissertation, Aerospace Engineering Department, Texas A\&M University, College Station, Texas, 2001.

${ }^{17}$ Bergh, H, Tijdeman, H., "Theoretical and experimental results for the dynamic response of pressure measuring systems,” NLR-TR F.238, 1965. 
${ }^{18}$ Steinle, F., Stanewsky, E., "Wind Tunnel Flow Quality and Data Accuracy Requirements." AGARD Advisory report 184, 1982.

${ }^{19}$ Walker, E.L., "Validation of Blockage Interference Correction in the National Transonic Facility (Invited)," AIAA Paper 2007-0750.

${ }^{20}$ Chan, D.T., et.el., "Mach Stability Improvements Using an Existing Second Throat Capability at the National Transonic Facility (Invited)," SciTech, January 2015.

${ }^{21}$ Rivers, S.M.," Comparisons of NTF, Ames and ETW results on the CRM,", SciTech, January 2015.

${ }^{22}$ Charles Bobbitt, J. and Everhart, J. L., "Status of the National Transonic Facility Characterization," AIAA Paper 2001-0755, AIAA, January 2001.

23 Kilgore, W.A., Balakrishna, S., and Butler, D.H., "Reduction of Tunnel Dynamics at the National Transonic Facility (Invited)", AIAA Paper 2001-1162, January 2001. 